\title{
Superconvergence and Reduced Integration in the Finite Element Method
}

\author{
By Miloš Zlámal
}

\begin{abstract}
The finite elements considered in this paper are those of the Serendipity family of curved isoparametric elements. There is given a detailed analysis of a superconvergence phenomenon for the gradient of approximate solutions to second order elliptic boundary value problems. An approach is proposed how to use the superconvergence in practical computations.
\end{abstract}

1. Introduction. Among finite elements the curved isoparametric elements of the Serendipity family (see Zienkiewicz [8]) are mostly used in the finite element codes prepared for engineering computations. It has been observed (see, e.g., Veryard [7], Irons and Razzaque [5], Barlow [1]) that applying quadratic members of this family a considerable improvement in accuracy of stresses is achieved if a reduced numerical integration-Gauss' $2 \times 2$ or $2 \times 2 \times 2$ product formulas-is used and the stresses are computed at Gaussian points, i.e. at points of these formulas. Here we want to analyze and justify this phenomenon. The results proved in the paper constitute a substantial extension of earlier results of the author [9].

We consider first the Dirichlet problem in two dimensions for a selfadjoint second order elliptic equation with variable coefficients as a model problem. We assume that the finite element partitions of the given domain are 2-strongly regular (see definition in the next section). In Section 4 we prove superconvergence of the gradient of the approximate solution at Gaussian points if Gauss' $2 \times 2$ formula for the two-dimensional cube $C_{2}:-1 \leqslant \xi_{i} \leqslant 1, i=1,2$, is applied. Numerical results (Section 6) indicate convincingly that superconvergence does not set in if the condition (2.8) about finite elements is not satisfied. Under a further assumption on finite elements the superconvergence is proved if there is applied any symmetric formula of the type (2.16) with positive coefficients which integrates exactly all polynomials from $\hat{Q}(3)$ on $C_{2}$ or any formula (2.16) which integrates exactly all polynomials from $\hat{P}(4)$ on $C_{2}(\hat{P}(k)$ and $\hat{Q}(k)$ denote the classes of polynomials of degree $k$ and of degree $k$ in each variable, respectively). This result shows that the superconvergence phenomenon is not closely connected with the reduced integration. However, Gauss' $2 \times 2$ formula has the smallest number of points among the above-mentioned formulas.

The theorem on superconvergence is true in three dimensions under the condition that the partitions are 3-strongly regular. In the last section there are introduced numerical results and an approach is proposed how to use the superconvergence in practical computations.

Received March 21, 1977; revised November 22, 1977.

AMS (MOS) subject classifications (1970). Primary 65N30, 65N15; Secondary 35A15.

Key words and phrases. Finite elements. 
2. Preliminaries. Let $\Omega$ be a bounded domain with a sufficiently smooth boundary $\Gamma$. We consider the Dirichlet problem

$$
\begin{aligned}
& L u=f(x) \quad \forall x \in \Omega,\left.\quad u\right|_{\Gamma}=0 \\
& L u=-\sum_{i, j=1}^{2} \frac{\partial}{\partial x_{i}}\left[a_{i j}(x) \frac{\partial u}{\partial x_{j}}\right]
\end{aligned}
$$

here $x=\left(x_{1}, x_{2}\right)$. Let us remark at this point that we could add a term $a_{0} u$ with $a_{0} \geqslant 0$ in the definition (2.1) of the operator $\mathrm{Lu}$. All that follows applies equally well to this case, with a straightforward supplementary analysis. To (2.1) there is associated the bilinear functional

$$
a(u, v)=\int_{\Omega} \sum_{i, j=1}^{2} a_{i j} \frac{\partial u}{\partial x_{i}} \frac{\partial v}{\partial x_{j}} d x .
$$

We assume that the coefficients are defined on $\bar{\Omega}$ and that

$$
a_{i j}(x)=a_{j i}(x), \quad \sum_{i, j=1}^{2} a_{i j}(x) \xi_{i} \xi_{j} \geqslant c_{1} \sum_{i=1}^{2} \xi_{i}^{2} \quad \forall x \in \Omega, c_{1}=\text { const }>0
$$

Hence $a(u, v)$ is $H_{0}^{1}(\Omega)$-elliptic.

The weak solution of the problem $(2.1)$ is a function $u \in H_{0}^{1}(\Omega)$ which satisfies

$$
a(u, v)=(f, v)_{0, \Omega} \quad \forall v \in H_{0}^{1}(\Omega) .
$$

We are using the usual notation for the Sobolev spaces:

$$
\begin{aligned}
H^{m}(\Omega) & =\left\{u \in L^{2}(\Omega), D^{\alpha} u \in L^{2}(\Omega) \forall|\alpha| \leqslant m\right\}, \quad m=0,1, \ldots, \\
H_{0}^{1}(\Omega) & =\left\{u \in H^{1}(\Omega),\left.u\right|_{\Gamma}=0\right\} .
\end{aligned}
$$

The norm in $H^{m}(\Omega)$ is denoted by $\|\cdot\|_{m, \Omega}$ and defined by

$$
\|u\|_{m, \Omega}=\left\{\sum_{|\alpha| \leqslant m}\left\|D^{\alpha} u\right\|_{L^{2}(\Omega)}^{2}\right\}^{1 / 2},
$$

the inner product in $H^{m}(\Omega)$ is denoted by $(\cdot, \cdot)_{m, \Omega}$. Often we shall use the seminorm

$$
|u|_{m, \Omega}=\left\{\sum_{|\alpha|=m}\left\|D^{\alpha} u\right\|_{L^{2}(\Omega)}^{2}\right\}^{1 / 2}
$$

(we set $|u|_{0, \Omega}=\|u\|_{0, \Omega}$ ).

To construct the finite element space $V_{h}$ in which the approximate solution will lie let us "cover" $\Omega$ by in general curved quadrilateral quadratic elements of the Serendipity family. Denote by $\hat{P}$ the class of incomplete cubic polynomials of the form

$$
\alpha_{1}+\alpha_{2} \xi_{1}+\alpha_{3} \xi_{2}+\alpha_{4} \xi_{1}^{2}+\alpha_{5} \xi_{1} \xi_{2}+\alpha_{6} \xi_{2}^{2}+\alpha_{7} \xi_{1}^{2} \xi_{2}+\alpha_{8} \xi_{1} \xi_{2}^{2}
$$

Evidently,

$$
\hat{P}(2) \subset \hat{P} \subset \hat{Q}(2) \text {. }
$$

Let $N_{j}\left(\xi_{1}, \xi_{2}\right)(j=1, \ldots, 8)$ be polynomials introduced in Zienkiewicz [8, p. 109] . Then $\Sigma_{j=1}^{8} v_{j} N_{j}\left(\xi_{1}, \xi_{2}\right)$ is the only polynomial from $\hat{P}$ assuming the given values $v_{j}$ 
at the nodes of the two-dimensional cube $C_{2}:-1 \leqslant \xi_{1} \leqslant 1,-1 \leqslant \xi_{2} \leqslant 1$, i.e. at the vertices and at the midpoints of the sides of $C_{2}$. This polynomial is a quadratic polynomial in each variable determined on every side of $C_{2}$ uniquely by its values at nodes of this side.

Now consider eight points (nodes) $a_{j}(j=1, \ldots, 8)$ with coordinates $\left(x_{1}^{j}, x_{2}^{j}\right)$ and the mapping

$$
x_{1}=x_{1}^{e}\left(\xi_{1}, \xi_{2}\right) \equiv \sum_{j=1}^{8} x_{1}^{j} N_{j}\left(\xi_{1}, \xi_{2}\right), \quad x_{2}=x_{2}^{e}\left(\xi_{1}, \xi_{2}\right) \equiv \sum_{j=1}^{8} x_{2}^{j} N_{j}\left(\xi_{1}, \xi_{2}\right) .
$$

If (2.7) maps the cube $C_{2}$ one-to-one on a closed domain $e$ lying in the $\left(x_{1}, x_{2}\right)$-plane, we call $e$ a quadratic quadrilateral element (curved or straight which depends on the choice of the nodes $a_{j}$ ).

We "cover" $\Omega$ by such elements, and we suppose that every "partition" of $\Omega$ by these elements is a 2 -strongly regular partition. By a $k$-strongly regular partition we understand a partition with the following properties:

(a) for every element the mapping (2.7) is a $C^{k+1}$ diffeomorphism (in particular, (2.7) is invertible).

(b) to every element $e$ there is associated a positive parameter $h_{e}$, and the mapping (2.7) is such that on $e$

$$
\begin{aligned}
& \left|D^{\alpha} x_{i}^{e}\right| \leqslant C_{1} h_{e}^{|\alpha|}, \quad|\alpha| \leqslant k+1, i=1,2, \\
& c_{2}^{-1} h_{e}^{2} \leqslant\left|J_{e}\right| \leqslant c_{2} h_{e}^{2}
\end{aligned}
$$

here $J_{e}\left(\xi_{1}, \xi_{2}\right)$ is the Jacobian of (2.7) and $C_{1}, c_{2}$ are positive constants independent of $h_{e}$ as well as of the chosen partition. If $h$ is defined by

$$
h=\max _{e} h_{e},
$$

then the constants $C_{1}, c_{2}$ are independent of $h$, too.

We will consider a family of 2 -strongly regular partitions of $\Omega$ such that $h \rightarrow 0$. We denote by $\Omega_{h}$ the interior of the union of all elements of the given partition (in general $\Omega_{h} \neq \Omega$ ); $\Gamma_{h}$ is its boundary.

Remark 1 . The definition of a $k$-strongly regular partition is similar to the definition of a $k$-regular family of elements by Ciarlet and Raviart [4]. The main difference is that, instead of their requirement $\left(2.17^{\prime}\right)$ (p. 427), we ask (2.8). This is evidently a much stronger condition, and every domain $\Omega$ cannot be covered by such elements. However, numerical results (see Section 6) indicate convincingly that (2.8) with $k=2$ is a necessary condition for superconvergence introduced later. In the following $\Omega$ is supposed to be such that there exists a family of 2-strongly regular partitions with $h \rightarrow 0$.

Remark 2. The following simple condition is sufficient for a partition to satisfy (2.8) and (2.9) for $h$ sufficiently small: to each element $e$ of the partition there exists a parallelogram $e^{\prime}$ with sides $h_{e}$ and $k_{e}, h_{e} \geqslant k_{e}$ (i.e., we denote the larger side by $h_{e}$ ), with angle $\omega_{e}$ and with nodes $a_{i}^{\prime}$ (the nodes corresponding to the midpoints of sides of $C_{2}$ must be midpoints of the sides of $e^{\prime}$ ) such that 


$$
\begin{aligned}
& \frac{k_{e}}{h_{e}} \geqslant c_{3}>0, \quad 0<\omega_{0} \leqslant \omega_{e} \leqslant \pi-\omega_{0}, \\
& \rho\left(a_{i}, a_{i}^{\prime}\right) \leqslant C_{2} h_{e}^{k+1}, \quad 1 \leqslant i \leqslant 8,
\end{aligned}
$$

where $\rho\left(a_{i}, a_{i}^{\prime}\right)$ is the distance of $a_{i}$ and $a_{i}^{\prime}$ and $\omega_{0}, c_{3}, C_{2}$ are positive constants independent of $h_{e}$, and the given partition, i.e. independent of $h$, too. To prove it, write $x_{i}^{e}=\sum_{j=1}^{8} x_{i}^{\prime j} N_{j}+\sum_{j=1}^{8}\left(x_{i}^{j}-x_{i}^{\prime j}\right) N_{j}\left(x_{1}^{\prime j}, x_{2}^{\prime j}\right.$ are coordinates of $\left.a_{j}^{\prime}\right)$. The mapping $x_{i}=\Sigma_{j=1}^{8} x_{i}^{\prime j} N_{j}\left(\xi_{1}, \xi_{2}\right)(i=1,2)$ is a mapping which maps $C_{2}$ on the parallelogram $e^{\prime}$ and midpoints on the midpoints of sides. Therefore, it is bilinear and we easily compute that $\partial x_{i}^{e^{\prime}} / \partial \xi_{j}$ are constant and bounded by $\left|\partial x_{i}^{e^{\prime}}\right| \partial \xi_{j} \mid \leqslant 1 / 2 h_{e}$ and $\left|J_{e^{\prime}}\right|$ $=1 / 4 h_{e} k_{e} \sin \omega_{e}$. Hence, $\left|D^{\alpha} x_{i}^{e^{\prime}}\right| \leqslant 1 / 2 h_{e}$ if $|\alpha|=1, D^{\alpha} x_{i}^{e^{\prime}}=0$, if $|\alpha| \geqslant 2$ and $\left(c_{3} / 4\right) \sin \omega_{0} h_{e}^{2} \leqslant\left|J_{e^{\prime}}\right| \leqslant 1 / 4 h_{e}^{2}$. From (2.11) it easily follows that (2.8) and (2.9) are true for $h_{e}$ sufficiently small.

Let us remark that the condition (b) is not as strong as (2.10) and (2.11) which effectively eliminate curved edges. E.g., consider a closed domain $\bar{\Omega}$ which is a map of a closed rectangle $\bar{R}$ and the corresponding mapping $x_{i}=\varphi_{i}\left(s_{1}, s_{2}\right), i=1,2$, is such that $\varphi_{i} \in C^{3}(\bar{R})$ and $\partial\left(\varphi_{1}, \varphi_{2}\right) / \partial\left(s_{1}, s_{2}\right) \neq 0$ on $\bar{R}$. We construct a mesh on $\bar{\Omega}$ in the following simple way: Its nodes are maps of nodes of a rectangular mesh of $\bar{R}$. Consider a rectangular element of $\bar{R}$ and denote by $h_{e}, k_{e}$, the lengths of its sides, $h_{e}$ being always the length of the larger one, and by $s_{1}^{0}, s_{2}^{0}$, the coordinates of its center. Let $e$ be the element of $\bar{\Omega}$ which corresponds to this rectangular element. Then one can easily express the functions $x_{i}^{e}$ from (2.7) and their Jacobian as follows (we may assume that $R$ has sides parallel to coordinate axes):

$$
\begin{aligned}
x_{i}^{e} \equiv & \sum_{j=1}^{8} x_{i}^{j} N_{j}\left(\xi_{1}, \xi_{2}\right)=\varphi_{i}\left(s_{1}^{0}, s_{2}^{0}\right)+\frac{1}{2} h_{e} \frac{\partial \varphi_{i}\left(s_{1}^{0}, s_{2}^{0}\right)}{\partial s_{1}} \xi_{1}+\frac{1}{2} k_{e} \frac{\partial \varphi_{i}\left(s_{1}^{0}, s_{2}^{0}\right)}{\partial s_{2}} \xi_{2} \\
& +\frac{1}{8} h_{e}^{2} \frac{\partial^{2} \varphi_{i}\left(s_{1}^{0}, s_{2}^{0}\right)}{\partial s_{1}^{2}} \xi_{1}^{2}+\frac{1}{4} h_{e} k_{e} \frac{\partial^{2} \varphi_{i}\left(s_{1}^{0}, s_{2}^{0}\right)}{\partial s_{1} \partial s_{2}} \xi_{1} \xi_{2}+\frac{1}{8} k_{e}^{2} \frac{\partial^{2} \varphi_{i}\left(s_{1}^{0}, s_{2}^{0}\right)}{\partial s_{2}^{2}} \xi_{2}^{2} \\
& +r_{i}\left(\xi_{1}, \xi_{2}\right), \quad D^{\alpha} r_{i}=O\left(h_{e}^{3}\right) \quad \text { for }|\alpha| \geqslant 0 . \\
J_{e}\left(\xi_{1}, \xi_{2}\right)= & \left.\frac{1}{4} h_{e} k_{e} \frac{\partial\left(\varphi_{1}, \varphi_{2}\right)}{\partial\left(s_{1}, s_{2}\right)}\right|_{s_{1}=s_{1}^{0}, s_{2}=s_{2}^{0}}+O\left(h_{e}^{3}\right) .
\end{aligned}
$$

Let us now assume that the rectangular mesh of $\bar{R}$ is chosen in such a way that $k_{e} / h_{e}$ $\geqslant c_{3}>0$ where $c_{3}$ is a positive constant independent of $h_{e}$ and the given mesh. Then the condition (b) is evidently satisfied for $k=2$. An example of the mapping $x_{i}=$ $\varphi_{i}\left(s_{1}, s_{2}\right)$ : polar coordinates.

Remark 3. The sign of $J_{e}$ changes if the local ordering of nodes is taken in the opposite direction. Therefore, we may and we will assume that for every $e$

$$
J_{e}\left(\xi_{1}, \xi_{2}\right)>0 \quad \forall \xi \in C_{2} .
$$

The functions $v$ from the finite element space $V_{h}$ are defined piecewise:

(2.13) $v\left(x_{1}, x_{2}\right)=\hat{v}\left[\xi_{1}^{e}\left(x_{1}, x_{2}\right), \xi_{2}^{e}\left(x_{1}, x_{2}\right)\right], \quad \hat{v}\left(\xi_{1}, \xi_{2}\right)=\sum_{j=1}^{8} v_{j} N_{j}\left(\xi_{1}, \xi_{2}\right)$. 
Here $\xi_{i}=\xi_{i}^{e}\left(x_{1}, x_{2}\right)$ is the inverse mapping to (2.7), and $v_{j}$ are values of $v$ at nodes of the element $e$. For the complete definition of $V_{h}$ it remains to ask $\left.v\right|_{\Gamma_{h}}=0$ which is equivalent to the requirement that the values of $v$ at nodes lying on $\Gamma$ are equal to zero. Evidently,

$$
V_{h} \subset C\left(\bar{\Omega}_{h}\right), \quad V_{h} \subset H_{0}^{1}\left(\Omega_{h}\right)
$$

To define the approximate solution of the problem (2.4) we proceed in a similar way as in [4]. We extend the solution $u \in H^{4}(\Omega)$ and the coefficients $a_{i j} \in H^{3}(\Omega)$ according to Calderon's extension theorem (see Nečas [6, p. 80]) to $R^{2}$ and denote these extensions by $\tilde{u}$ and $\tilde{a}_{i j}$, respectively. We also extend $f$ as follows:

$$
\tilde{f}=-\sum_{i, j=1}^{2} \frac{\partial}{\partial x_{i}}\left(\tilde{a_{i j}} \frac{\partial \tilde{u}}{\partial x_{j}}\right) \in H^{2}(\Omega) .
$$

Denote by $\tilde{a}(w, v)$ the bilinear functional $\int_{\Omega_{h}} \Sigma_{i, j=1}^{2} \tilde{a}_{i j}\left(\partial w / \partial x_{i}\right)\left(\partial v / \partial x_{j}\right) d x$. Due to $\left.v\right|_{\Gamma_{h}}=0$ we get for any $v \in V_{h}$ by Green's theorem $\tilde{a}(\tilde{u}, v)=(\widetilde{f, v})_{0, \Omega_{h}}$. For simplicity of writing we will leave out the sign $\sim$ and write

$$
\begin{gathered}
a(u, v)=(f, v)_{0, \Omega_{h}} \quad \forall v \in V_{h}, \\
a(u, v)=\int_{\Omega_{h}} \sum_{i, j=1}^{2} a_{i j} \frac{\partial u}{\partial x_{i}} \frac{\partial v}{\partial x_{j}} d x .
\end{gathered}
$$

This will not cause any confusion in the estimates carried out later. All constants will depend on $\|\tilde{u}\|_{4, \Omega_{h}}$ and $\|\tilde{f}\|_{2, \Omega_{h}}$. The first norm is bounded, according to Calderon's theorem, by $\|u\|_{4, \Omega}$. Evidently, also $\|\widetilde{f}\|_{2, \Omega_{h}}$ is bounded by this norm. By (2.3) the matrix $A=\left\{a_{i j}\right\}_{i, j=1}^{2}$ is uniformly positive definite for $x \in \Omega_{h}$ and $h$ sufficiently small if the extensions of the coefficients are continuous. Hence, under this condition

$$
\sum_{i, j=1}^{2} a_{i j}(x) \xi_{i} \xi_{j} \geqslant c_{1} \sum_{i=1}^{2} \xi_{i}^{2} \forall x \in \Omega_{h},
$$

where $c_{1}$ is a positive constant independent on $h$.

We could define the approximate solution $u_{h}$ as that function from $V_{h}$ which satisfies $a\left(u_{h}, v\right)=(f, v)_{0, \Omega_{h}} v \in V_{h}$. However, in general the values of $a(w, v)$ and $(f, v)_{0, \Omega_{h}}$ for $v, w \in V_{h}$ cannot be computed exactly. Numerical integration is the usual and only practical way out. To this end let us consider quadrature formulas $I(\varphi)$ for the cube $C_{2}$ of the form

$$
I(\varphi)=\sum_{r} A_{r} \varphi\left(Q_{r}\right)
$$

We make the assumption that the points $Q_{r}$ of the formula belong to the interior of $C_{2}$ or are nodes of $C_{2}$. Then expressing $a(w, v)$ as a sum of integrals over the elements $e$, transforming these integrals by means of (2.7) in integrals over $C_{2}$ and using (2.16), we get the approximate value $a_{h}(w, v)$ of $a(w, v)$ :

$$
a_{h}(w, v)=\sum_{e} \sum_{r} A_{r} J_{e}\left(Q_{r}\right) \sum_{i, j=1}^{2} \hat{a}_{i j}\left(Q_{r}\right) \frac{\widehat{\partial w}}{\partial x_{i}}\left(Q_{r}\right) \frac{\widehat{\partial v}}{\partial x_{j}}\left(Q_{r}\right)
$$


Here the following notation (in agreement with the notation in (2.13)) is used for any function $g$ defined on $\bar{\Omega}_{h}$ :

$$
\hat{g}\left(\xi_{1}, \xi_{2}\right)=g\left[x_{1}^{e}\left(\xi_{1}, \xi_{2}\right), x_{2}^{e}\left(\xi_{1}, \xi_{2}\right)\right]
$$

Similarly,

$$
f_{h}(v)=\sum_{e} \sum_{r} A_{r} J_{e}\left(Q_{r}\right) \hat{f}\left(Q_{r}\right) \hat{v}\left(Q_{r}\right)
$$

is the approximate value of $(f, v)_{0, \Omega_{h}}$. Our assumption concerning the points $Q_{r}$ guarantees that, at least for $h$ sufficiently small, we do not need for the computation of $a_{h}(w, v)$ and $f_{h}(v)$ values of data at other points than at points from $\bar{\Omega}$. Now the approximate solution $u_{h} \in V_{h}$ is defined by

$$
a_{h}\left(u_{h}, v\right)=f_{h}(v) \quad \forall v \in V_{h} .
$$

It is clear from the remark made above that $u_{h}$ does not depend on extensions of the coefficients $a_{i j}$ and the right-hand side $f$ of the equation (2.1). In general, it is not true that $u_{h}$ exists and is unique. We will consider the cases that $I(\varphi)$ is Gauss' product formula $2 \times 2$ or any symmetric formula with positive coefficients which integrates exactly all polynomials from $\hat{Q}(3)$ (Gauss' $2 \times 2$ is a special case of such formulas having the smallest number of points) or any formula which integrates exactly all polynomials from $\hat{P}(4)$. The existence and uniqueness of $u_{h}$ will follow from Lemma 3.6.

3. Some Lemmas. In what follows we denote by $C$ a generic positive constant not necessarily the same in any two places which does not depend on $h_{e}, h$ and on some functions. It will be clear from the context of which functions the constant is independent.

LEMma 3.1. We have for any $\hat{v} \in \hat{P}$

$$
\begin{gathered}
|\hat{v}|_{j, C_{2}} \leqslant C|\hat{v}|_{i, C_{2}}, \quad 0 \leqslant i<j \leqslant 3\left(|\hat{v}|_{0, C_{2}} \equiv\|\hat{v}\|_{0, C_{2}}\right), \\
\max _{C_{2}}\left|D^{\alpha} \hat{v}\right| \leqslant C|\hat{v}|_{|\alpha|, C_{2}}, \quad|\alpha| \leqslant 3 .
\end{gathered}
$$

Proof. To prove (3.1) for $j=1$ it is sufficient to realize that $\|\hat{v}\|_{0, C_{2}}^{2}$ is a positive definite quadratic form of the coefficients $\alpha_{j}(j=1, \ldots, 8)$ and $|\hat{v}|_{1, c_{2}}^{2}$ is a bounded quadratic form of these coefficients. Applying (3.1) with $j=1$ to partial derivatives of $\hat{v}$ we get (3.1) for $j=2,3$. (3.2) follows from equivalence of all norms of finite dimensional spaces.

LemMA 3.2. Let $g \in H^{i}\left(\Omega_{h}\right), 0 \leqslant i \leqslant 3$. Then

$$
|\hat{g}|_{i, C_{2}} \leqslant C h_{e}^{i-1}\|g\|_{i, e} .
$$

Proof. We transform the integral $|\hat{g}|_{i, C_{2}}^{2}$ by means of the inverse mapping of the mapping (2.7). (3.3) follows from (2.8) and (2.9) (the Jacobian $J_{e}^{-1}$ of the inverse mapping is bounded by $c_{2} h_{e}^{-2}$ ).

Often, we shall make use of the Bramble-Hilbert lemma (see [2] and [3]) on linear functionals. In fact, this lemma will be applied for the domain $C_{2}$ only. 
LEMma 3.3 (SPECiAl CASE of THE BRAMble-Hilbert lemma). Let the linear functional $L(\varphi)$ be bounded on $H^{k+1}\left(C_{2}\right),|L(\varphi)| \leqslant M\|\varphi\|_{k+1, C_{2}}$, and let it vanish for $\varphi \in \hat{P}(k)$. Then there exists a constant $C$ independent on $\varphi$ such that

$$
|L(\varphi)| \leqslant C M|\varphi|_{k+1, C_{2}} \quad \forall \varphi \in H^{k+1}\left(C_{2}\right) .
$$

If $L(\varphi)$ vanishes for $\varphi \in \hat{Q}(k)$, then

$$
|L(\varphi)| \leqslant C M\left\{\left\|\frac{\partial^{k+1} \varphi}{\partial \xi_{1}^{k+1}}\right\|_{0, C_{2}}+\left\|\frac{\partial^{k+1} \varphi}{\partial \xi_{2}^{k+1}}\right\|_{0, C_{2}}\right\} .
$$

Lemma 3.3 allows us to estimate the interpolation error for a given function. The interpolate $\varphi_{I}$ of a function $\varphi$ defined on $C_{2}$ is the polynomial $\Sigma_{j=1}^{8} \varphi_{j} N_{j}\left(\xi_{1}, \xi_{2}\right)$, where $\varphi_{j}$ are the values of $\varphi$ at the nodes of $C_{2}$. The interpolate $g_{I}$ of a function $g$ defined on $\bar{\Omega}_{h}$ is the function from $V_{h}$ which assumes the same values at all nodes of the given partition as the function $g$.

LEMMA 3.4. If $\varphi \in H^{3}\left(C_{2}\right)$, then

$$
\left\|\varphi-\varphi_{I}\right\|_{j, C_{2}} \leqslant\left. C ! \varphi\right|_{3, C_{2}}, \quad j=0, \ldots, 3 .
$$

Proof. We get (3.6) if we apply Lemma 3.3 to the functional $L(\varphi)=$ $\left(\varphi-\varphi_{I}, w\right)_{j, C_{2}}$, and afterwards we set $\varphi-\varphi_{I}$ for $w$.

We shall need estimates of the error functional $E(\varphi)=\int_{C_{2}} \varphi d \xi-I(\varphi)$. Such estimates follow immediately from (3.5) and (3.4).

LEMMA 3.5. Let $I(\varphi)$ be a formula which integrates exactly all polynomials from Q(3). Then

$$
|E(\varphi)| \leqslant C\left\{\left\|\frac{\partial^{4} \varphi}{\partial \xi_{1}^{4}}\right\|_{0, C_{2}}+\left\|\frac{\partial^{4} \varphi}{\partial \xi_{2}^{4}}\right\|_{0, C_{2}}\right\} .
$$

If $I(\varphi)$ integrates exactly all polynomials from $\hat{P}(4)$, then

$$
|E(\varphi)| \leqslant C|\varphi|_{5, C_{2}} .
$$

The following is the main lemma from which, among other things, existence and uniqueness of the approximate solution $u_{h}$ follows.

LEMMA 3.6. Let $I(\varphi)$ be any symmetric formula with positive coefficients which integrates exactly all polynomials from $\hat{Q}(3)$ or any formula which integrates exactly all polynomials from $\hat{P}(4)$. Let the coefficients $a_{i j}$ satisfy $\left(2.3^{\prime}\right)$ and let them be bounded and in the latter case be Lipschitz continuous on $\bar{\Omega}_{h}$. Finally, let the finite element partitions be 1-strongly regular (in fact, it is sufficient that (2.8) be true for $|\alpha| \leqslant 1$ and $|\alpha| \leqslant 2$, respectively). Then $|v|_{h}=\left\{a_{h}(v, v)\right\}^{1 / 2}$ is a norm on $V_{h}$ equivalent uniformly with respect to $h$ to the norm $|v|_{1, \Omega_{h}}$, i.e. there exists a constant $c_{4}$ independent of $h$ such that

$$
c_{4}^{-1}|v|_{1, \Omega_{h}} \leqslant|v|_{h} \leqslant c_{4}|v|_{1, \Omega_{h}} \quad \forall v \in V_{h} .
$$

Remark 4. Among formulas satisfying the assumptions of Lemma 3.6 Gauss' $2 \times 2$ formula has the smallest number of points. 
Proof. (a) Let $I(\varphi)$ be a symmetric formula with positive coefficients which integrates exactly all polynomials from $\hat{Q}(3)$. Denote by $I^{*}(\varphi)$ the special case of Gauss' $2 \times 2$ formula. Denote by $\gamma$ the value $\gamma=I\left(\xi_{1}^{4}\right)=I\left(\xi_{2}^{4}\right)$ and by $\alpha$ the value $\alpha=(45 / 16)(4 / 5-\gamma)$. As $I^{*}\left(\xi_{1}^{4}\right)=I^{*}\left(\xi_{2}^{4}\right)=4 / 9$, we easily find that if $\alpha \neq 1$, the formula

$$
I^{0}(\varphi)=\frac{1}{1-\alpha}\left[I(\varphi)-\alpha I^{*}(\varphi)\right]
$$

integrates exactly all polynomials from $\hat{P}(4)$. Hence

$$
I(\varphi)=\alpha I^{*}(\varphi)+(1-\alpha) I^{0}(\varphi) .
$$

If $\alpha=1$, then $I\left(\xi_{i}^{4}\right)=I^{*}\left(\xi_{i}^{4}\right)(i=1,2)$, and $E^{1}(\varphi)=I(\varphi)-I^{*}(\varphi)$ satisfies (3.8). We have

$$
I(\varphi)=I^{*}(\varphi)+E^{1}(\varphi)
$$

Now consider the function $\psi=\left(\partial \hat{v} / \partial \xi_{1}\right)^{2}+\left(\partial \hat{v} / \partial \xi_{2}\right)^{2}$, where $\hat{v} \in \hat{P}$. As $\psi \in$ $\hat{P}(4)$ it follows from (3.10) and (3.11), respectively, that $I(\psi)=\alpha I^{*}(\psi)+$ $(1-\alpha) \int_{C_{2}} \psi d \xi$. As $\hat{v}$ is of the form (2.5), $\int_{C_{2}} \psi d \xi$ must be of the form $\mathrm{z}^{T} A \mathrm{z}$ where $\mathrm{z}=\left(\alpha_{2}, \ldots, \alpha_{8}\right)^{T}$ and $A$ is a symmetric $7 \times 7$ matrix. Further, we compute easily $I^{*}(\psi)=\mathrm{z}^{T} A \mathrm{z}-(16 / 45)\left(\alpha_{7}^{2}+\alpha_{8}^{2}\right)$; hence

$$
I(\psi)=\mathrm{z}^{T} A \mathrm{z}-\frac{16}{45} \alpha\left(\alpha_{7}^{2}+\alpha_{8}^{2}\right)=\left(1-\frac{4}{9} \alpha\right) \mathrm{z}^{T} A \mathrm{z}+\frac{4}{9} \alpha\left[\mathrm{z}^{T} A \mathrm{z}-\frac{4}{5}\left(\alpha_{7}^{2}+\alpha_{8}^{2}\right)\right] .
$$

A direct computation gives

$$
\begin{aligned}
\mathrm{z}^{T} A \mathrm{z}-\frac{4}{5}\left(\alpha_{7}^{2}+\alpha_{8}^{2}\right)= & 4\left(\alpha_{2}^{2}+\frac{2}{3} \alpha_{2} \alpha_{8}+\frac{4}{9} \alpha_{8}^{2}\right)+4\left(\alpha_{3}^{2}+\frac{2}{3} \alpha_{3} \alpha_{7}+\frac{4}{9} \alpha_{7}^{2}\right) \\
& +\frac{16}{3} \alpha_{4}^{2}+\frac{8}{3} \alpha_{5}^{2}+\frac{16}{3} \alpha_{6}^{2} \geqslant 0 .
\end{aligned}
$$

As $\gamma$ is always positive, $\alpha$ must be smaller than $9 / 4$; and setting $c=1-4 \alpha / 9>0$, we have

$$
I(\psi) \equiv I\left(\left(\frac{\partial \hat{v}}{\partial \xi_{1}}\right)^{2}+\left(\frac{\partial \hat{v}}{\partial \xi_{2}}\right)^{2}\right) \geqslant c \int_{C_{2}} \psi d \xi \equiv c|\hat{v}|_{1, C_{2}}^{2} \quad \forall \hat{v} \in \hat{P}
$$

This inequality will be used to prove the first part of Lemma 3.6.

(b) From (2.3'), (2.12), (2.17) (the coefficients $A_{r}$ are positive) we get

$$
a_{h}(v, v) \geqslant c_{1} \sum_{e} I\left(J_{e}\left[\left(\frac{\partial \vec{v}}{\partial x_{1}}\right)^{2}+\left(\frac{\partial v}{\partial x_{2}}\right)^{2}\right]\right) .
$$

If $\Delta_{x}$ is the vector $\left(\widehat{\partial v} / \partial x_{1}, \widehat{\partial v} / \partial x_{2}\right)^{T}$ and $\Delta_{\xi}$ the vector $\left(\partial \hat{v} / \partial \xi_{1}, \partial \hat{v} / \partial \xi_{2}\right)^{T}$, we have $\Delta_{\xi}=D \Delta_{x}$ where $D=\left\{\partial x_{j}^{e} / \partial \xi_{i}\right\}_{i, j=1}^{2}$. From (2.8) it follows $\|D\|^{2} \leqslant C h_{e}^{2}$. If we compute $D^{-1}$ and take into account (2.9), we get $\left\|D^{-1}\right\|^{2} \leqslant C h_{e}^{-2}$. Now for any nonsingular matrix $M$ the matrix $M^{T} M$ is positive definite and $\Delta_{\xi}^{T} M^{T} M \Delta_{\xi} \geqslant$ $\left\|M^{-1}\right\|^{-2}\left\|\Delta_{\xi}\right\|^{2}$. Therefore,

$$
\begin{aligned}
J_{e}\left[\left(\frac{\partial v}{\partial x_{1}}\right)^{2}+\left(\frac{\widehat{\partial v}}{\partial x_{2}}\right)^{2}\right] & =J_{e}\left\|\Delta_{x}\right\|^{2}=J_{e} \Delta_{\xi}^{T}\left(D^{-1}\right)^{T} D^{-1} \Delta_{\xi} \geqslant J_{e}\|D\|^{-2}\left\|\Delta_{\xi}\right\|^{2} \\
& \geqslant C\left\|\Delta_{\xi}\right\|^{2}=C \psi ;
\end{aligned}
$$


and with respect to (3.13) and (3.12), $a_{h}(v, v) \geqslant c_{1} C \Sigma_{e} I(\psi) \geqslant C \Sigma_{e}|\hat{v}|_{1, C_{2}}^{2}$.

On the other hand, $\left\|\Delta_{\xi}\right\|^{2}=\Delta_{x}^{T} D^{T} D \Delta_{x} \geqslant\left\|D^{-1}\right\|^{-2}\left\|\Delta_{x}\right\|^{2} \geqslant C h_{e}^{2}\left\|\Delta_{x}\right\|^{2}$; hence

$$
|\hat{v}|_{1, C_{2}}^{2} \geqslant C h_{e}^{2} \int_{e} J_{e}^{-1}\left[\left(\frac{\partial v}{\partial x_{1}}\right)^{2}+\left(\frac{\partial v}{\partial x_{2}}\right)^{2}\right] d x \geqslant C \int_{e}\left[\left(\frac{\partial v}{\partial x_{1}}\right)^{2}+\left(\frac{\partial v}{\partial x_{2}}\right)^{2}\right] d x
$$

i.e. $|\hat{v}|_{1, C_{2}}^{2} \geqslant C|v|_{1, e}^{2} \forall \hat{v} \in \hat{P}$, and the final estimate is $a_{h}(v, v) \geqslant C \Sigma_{e}|v|_{1, e}^{2}=C|v|_{1, \Omega_{h}}^{2}$.

(c) Let $I(\varphi)$ integrate exactly all polynomials from $\hat{P}(4)$. Consider first the sum

$$
S_{e}=J_{e} \sum_{i, j=1}^{2} \hat{a}_{i j} \frac{\widehat{\partial v}}{\partial x_{i}} \frac{\widehat{\partial v}}{\partial x_{j}}
$$

(the values of this sum at $Q_{r}$ appear in (2.17)). We have

$$
S_{e}=\Delta_{\xi}^{T} B \Delta_{\xi}=\sum_{i, j=1}^{2} b_{i j} \frac{\partial \hat{v}}{\partial \xi_{i}} \frac{\partial \hat{v}}{\partial \xi_{j}}, \quad B=J_{e}\left(D^{-1}\right)^{T} \hat{A} D^{-1}
$$

( $\hat{A}$ is the matrix $\left\{\hat{a}_{i j}\right\}_{i, j=1}^{2}$ ). Elementary computations give the following expressions for the coefficients of the symmetric matrix $B$ :

$$
\begin{aligned}
& b_{11}=J_{e}^{-1}\left\{\left(\frac{\partial x_{2}^{e}}{\partial \xi_{2}}\right)^{2} \hat{a}_{11}-2 \frac{\partial x_{1}^{e}}{\partial \xi_{2}} \frac{\partial x_{2}^{e}}{\partial \xi_{2}} \hat{a}_{12}+\left(\frac{\partial x_{1}^{e}}{\partial \xi_{2}}\right)^{2} \hat{a}_{22}\right\}, \\
& b_{12}=J_{e}^{-1}\left\{-\frac{\partial x_{2}^{e}}{\partial \xi_{1}} \frac{\partial x_{2}^{e}}{\partial \xi_{2}} \hat{a}_{11}+\left(\frac{\partial x_{1}^{e}}{\partial \xi_{1}} \frac{\partial x_{2}^{e}}{\partial \xi_{2}}+\frac{\partial x_{2}^{e}}{\partial \xi_{1}} \frac{\partial x_{1}^{e}}{\partial \xi_{2}}\right) \hat{a}_{12}-\frac{\partial x_{1}^{e}}{\partial \xi_{1}} \frac{\partial x_{1}^{e}}{\partial \xi_{2}} \hat{a}_{22}\right\}, \\
& b_{22}=J_{e}^{-1}\left\{\left(\frac{\partial x_{2}^{e}}{\partial \xi_{1}}\right)^{2} \hat{a}_{11}-2 \frac{\partial x_{1}^{e}}{\partial \xi_{1}} \frac{\partial x_{2}^{e}}{\partial \xi_{1}} \hat{a}_{12}+\left(\frac{\partial x_{1}^{e}}{\partial \xi_{1}}\right)^{2} \hat{a}_{22}\right\} .
\end{aligned}
$$

Let us denote by $\beta$ any of the factors appearing at any of the coefficients $\hat{a}_{i j}$ on the right-hand sides of (3.15). We shall need later the following estimate of $\beta$ :

$$
\left|D^{\alpha} \beta\right| \leqslant C h_{e}^{|\alpha|}, \quad|\alpha| \geqslant 0
$$

(to prove (3.16) differentiate the identity $J_{e} J_{e}^{-1}=1$ and prove by induction $D^{\alpha} J_{e}^{-1}=$ $O\left(h_{e}^{-2+|\alpha|}\right)$; (3.16) follows by Leibniz rule). At this time we use (3.16) with $|\alpha| \leqslant 1$.

Lipschitz continuity of $a_{i j}$ and (3.16) with $|\alpha| \leqslant 1$ give $b_{i j}=b_{i j}^{0}+O\left(h_{e}\right)$ where $b_{i j}^{0}$ means the value of $b_{i j}$ at the center $(0,0)$. Therefore

$$
S_{e}=\Delta_{\xi}^{T} B^{0} \Delta_{\xi}+O\left(h_{e}\right)\left\|\Delta_{\xi}\right\|^{2}=\sum_{i, j=1}^{2} b_{i j}^{0} \frac{\partial \hat{v}}{\partial \xi_{i}} \frac{\partial \hat{v}}{\partial \xi_{j}}+O\left(h_{e}\right)|\hat{v}|_{1, C_{2}}^{2}, \quad B^{0}=\left\{b_{i j}^{0}\right\}_{i, j=1}^{2} .
$$

As $\left(\partial \hat{v} / \partial \xi_{i}\right)\left(\partial \hat{v} / \partial \xi_{j}\right) \in \ddot{P}(4)$, we get

$$
\begin{gathered}
\sum_{r} A_{r} J_{e}\left(Q_{r}\right) \sum_{i, j=1}^{2} \hat{a}_{i j}\left(Q_{r}\right) \frac{\widehat{\partial v}}{\partial x_{i}}\left(Q_{r}\right) \frac{\widehat{\partial v}}{\partial x_{j}}\left(Q_{r}\right) \\
=\sum_{r} A_{r} \sum_{i, j=1}^{2} b_{i j}^{0} \frac{\partial \hat{v}\left(Q_{r}\right)}{\partial \xi_{i}} \frac{\partial \hat{v}\left(Q_{r}\right)}{\partial \xi_{j}}+O\left(h_{e}\right)|\hat{v}|_{1, C_{2}}^{2} \\
=\int_{C_{2}} \sum_{i, j=1}^{2} b_{i j}^{0} \frac{\partial \hat{v}}{\partial \xi_{i}} \frac{\partial \hat{v}}{\partial \xi_{j}} d \xi+O\left(h_{e}\right)|\hat{v}|_{1, C_{2}}^{2} .
\end{gathered}
$$


Further, $\Delta_{\xi}^{T} B \Delta_{\xi} \geqslant c_{1} J_{e} \Delta_{\xi}^{T}\left(D^{-1}\right)^{T} D^{-1} \Delta_{\xi}$ (because $B=J_{e}\left(D^{-1}\right)^{T} \hat{A} D^{-1}$ and $A$ satisfies $\left(2.3^{\prime}\right)$ ). We have proved before that $J_{e} \Delta_{\xi}^{T}\left(D^{-1}\right)^{T} D^{-1} \Delta_{\xi} \geqslant C\left\|\Delta_{\xi}\right\|^{2}$. Therefore,

$$
\Delta_{\xi}^{T} B^{0} \Delta_{\xi} \geqslant C\left[\left(\frac{\partial \hat{v}}{\partial \xi_{1}}\right)^{2}+\left(\frac{\partial \hat{v}}{\partial \xi_{2}}\right)^{2}\right] \text { and } \int_{C_{2}} \sum_{i, j=1}^{2} b_{i j}^{0} \frac{\partial \hat{v}}{\partial \xi_{i}} \frac{\partial \hat{v}}{\partial \xi_{j}} d \xi \geqslant C|\hat{v}|_{1, C_{2}}^{2} \text {. }
$$

Consequently, for $h$ sufficiently small,

$$
\begin{aligned}
a_{h}(v, v) & =\sum_{e} \sum_{r} A_{r} J_{e}\left(Q_{r}\right) \sum_{i, j=1}^{2} \hat{a}_{i j}\left(Q_{r}\right) \frac{\widehat{\partial v}}{\partial x_{i}}\left(Q_{r}\right) \frac{\widehat{\partial v}}{\partial x_{j}}\left(Q_{r}\right) \\
& \geqslant C \sum_{e}|\hat{v}|_{1, C_{2}}^{2} \geqslant C \sum_{e}|v|_{1, e}^{2}=C|v|_{1, \Omega_{h}}^{2} .
\end{aligned}
$$

(d) We have

$$
a_{h}(v, v)=\sum_{e} \sum_{r} A_{r} \sum_{i, j=1}^{2} b_{i j}\left(Q_{r}\right) \frac{\partial \hat{v}\left(Q_{r}\right)}{\partial \xi_{i}} \frac{\partial \hat{v}\left(Q_{r}\right)}{\partial \xi_{j}} .
$$

As $\beta$ and $\hat{a}_{i j}$ are bounded, so are bounded the coefficients $b_{i j}$. Hence, from (3.2) and (3.3) we easily get $a_{h}(v, v) \leqslant C \Sigma_{e}|\hat{v}|_{1, C_{2}}^{2} \leqslant C|v|_{1, \Omega_{h}}^{2}$.

4. Superconvergence Theorems. First, the integration by Gauss' $2 \times 2$ formula is considered. Besides the special notation $I^{*}(\varphi)$, for this formula we will use the sign * for other quantities as, e.g., for $u_{h}^{*}, a_{h}^{*}(v, v), f_{h}^{*}(v),|\cdot|_{h}^{*}, E^{*}, Q_{r}^{*}$. The rate of convergence will not be expressed by means of the norm $|\cdot|_{h}^{*}$, because it depends on the coefficients $a_{i j}$ of the operator $L u$. We consider the norm $|\cdot|_{h}^{*}$ associated to the operator $L u=-\Delta u$ and this norm is denoted by $\|\cdot\|_{h}$. Hence, $\left(A_{r}^{*}\right.$ are equal to 1$)$

$$
\begin{gathered}
\|v\|_{h}=\left\{\sum_{e} \sum_{r=1}^{4} J_{e}\left(Q_{r}^{*}\right)\left[\left(\frac{\widehat{\partial v}}{\partial x_{1}}\left(Q_{r}^{*}\right)\right)^{2}+\left(\frac{\partial \widehat{\partial v}}{\partial x_{2}}\left(Q_{r}^{*}\right)\right)^{2}\right]\right\}^{3 / 2}, \\
Q_{r}^{*}=\left( \pm \frac{\sqrt{3}}{3}, \pm \frac{\sqrt{3}}{3}\right) .
\end{gathered}
$$

The norm $\|\cdot\|_{h}$ is on $V_{h}$ equivalent uniformly with respect to $h$ to the norm $|v|_{1, \Omega_{h}}$ :

$$
c_{4}^{-1}|v|_{1, \Omega_{h}} \leqslant\|v\|_{h} \leqslant c_{4}|v|_{1, \Omega_{h}} \quad \forall v \in V_{h} .
$$

Ciarlet and Raviart proved (see [4, p. 462]) the following estimate for the discretization error $u-u_{h}$ where $u$ is the solution of (2.4) and $u_{h}$ the solution of (2.19):

$$
\left\|u-u_{h}\right\|_{1, \Omega_{h}} \leqslant C h^{2}
$$

(they consider 9 degrees of freedom elements; however the bound can be proved in the same way for 8 degrees of freedom elements considered here). We can say that in the sense of $L_{2}$-norm average error of the gradient is of the order $O\left(h^{2}\right)$. We shall prove that $\left\|u-u_{h}^{*}\right\|_{h} \leqslant C h^{3}$, and this is the reason that we speak about superconvergence. In fact, let us denote by $N_{G}$ the number of all Gaussian points and by $E(P)$ the error of the gradient, 


$$
E(P)=\left[\left(\frac{\partial\left(u-u_{h}^{*}\right)(P)}{\partial x_{1}}\right)^{2}+\left(\frac{\partial\left(u-u_{h}^{*}\right)(P)}{\partial x_{2}}\right)^{2}\right]^{1 / 2}
$$

We have

$$
\text { meas } \Omega_{h}=\sum_{e} \int_{e} d x=\sum_{e} \int_{C_{2}} J_{e} d \xi \leqslant C h^{2} N_{G}
$$

therefore, $N_{G} \geqslant C h^{-2}, C>0$. By the Cauchy inequality we prove under the additional assumption $h_{e} / h \geqslant C>0 \forall e$

$$
N_{G}^{-1} \sum_{P \in G} E(P) \leqslant C\left\|u-u_{h}^{*}\right\|_{h} .
$$

Hence, it follows that the arithmetic mean of errors of the gradient at Gaussian points is $O\left(h^{3}\right)$.

THEOREM 4.1. Let the finite element partitions of $\Omega$ be 2-strongly regular. Further, assume the boundary $\Gamma$ to be sufficiently smooth,

$$
u \in H^{4}(\Omega), \quad a_{i j} \in H^{3}(\Omega), \quad f \in H^{3}(\Omega),
$$

and the operator Lu to be uniformly elliptic. Finally, let the quadrature formula (2.16) be Gauss' $2 \times 2$ product formula. Then there exists a constant $C$ independent on $h$ (it is of the form $C_{1}\|u\|_{4, \Omega}+C_{2}\|f\|_{3, \Omega}$ where $C_{1}$ and $C_{2}$ do not depend both on $h$ and $u$ and $f$ ) such that

$$
\left\|u-u_{h}^{*}\right\|_{h} \leqslant C h^{3} .
$$

Proof. (a) Subtracting (2.19) from (2.15), we get

$$
a_{h}^{*}\left(u-u_{h}^{*}, v\right)=S_{h}^{*}(v)-R_{h}^{*}(u, v) \quad \forall v \in V_{h},
$$

where

$$
R_{h}^{*}(u, v)=a(u, v)-a_{h}^{*}(u, v), \quad S_{h}^{*}(v)=(f, v)_{0, \Omega_{h}}-f_{h}^{*}(v)
$$

Further,

$$
a_{h}^{*}\left(u_{I}-u_{h}^{*}, v\right)=S_{h}^{*}(v)-R_{h}^{*}(u, v)-a_{h}^{*}\left(u-u_{I}, v\right) \quad \forall v \in V_{h}
$$

( $u_{I}$ is the interpolate of $u$ ). We prove later that

$$
\left.\begin{array}{r}
\left|a_{h}^{*}\left(u-u_{I}, v\right)\right| \leqslant C h^{3}|v|_{1, \Omega_{h}} \\
\left|R_{h}^{*}(u, v)\right| \leqslant C h^{3}|v|_{1, \Omega_{h}} \\
\left|S_{h}^{*}(v)\right| \leqslant C h^{3}|v|_{1, \Omega_{h}}
\end{array}\right\} \quad \forall v \in V_{h} .
$$

From these inequalities, (4.7) and (3.9) it follows that

$$
\left|a_{h}^{*}\left(u_{I}-u_{h}^{*}, v\right)\right| \leqslant C h^{3}|v|_{h}^{*} \quad \forall v \in V_{h} .
$$

Setting $v=u_{I}-u_{h}^{*} \in V_{h}$, we obtain

$$
\left|u_{I}-u_{h}^{*}\right|_{h}^{*} \leqslant C h^{3}
$$


Consequently, $\left|u-u_{h}^{*}\right|_{h}^{*} \leqslant\left|u-u_{I}\right|_{h}^{*}+\left|u_{I}-u_{h}^{*}\right|_{h}^{*} \leqslant\left|u-u_{I}\right|_{h}^{*}+C h^{3}$. We also prove

$$
\left|u-u_{I}\right|_{h}^{*} \leqslant C h^{3} \text {. }
$$

The last two inequalities give

$$
\left|u-u_{h}^{*}\right|_{h}^{*} \leqslant C h^{3} .
$$

Now from (4.1), (2.17) (with $\left.A_{r}=1, Q_{r}=Q_{r}^{*}, r=1, \ldots, 4\right)$ and (2.3') it follows for any function $g$ piecewise differentiable in $\Omega_{h}$ that

$$
\|g\|_{h} \leqslant C|g|_{h}^{*}
$$

which together with (4.14) proves the theorem.

(b) We prove (4.8) and (4.13). We express $a_{h}^{*}(\omega, v)$, where $v \in V_{h}$ and $\omega$ is any function such that $a_{h}^{*}(\omega, v)$ is defined, as follows (see (3.14)):

$$
a_{h}^{*}(\omega, v)=\sum_{e} \sum_{r=1}^{4} \sum_{i, j=1}^{2} b_{i j}\left(Q_{r}^{*}\right) \frac{\partial \hat{\omega}\left(Q_{r}^{*}\right)}{\partial \xi_{i}} \frac{\partial \hat{v}\left(Q_{r}^{*}\right)}{\partial \xi_{j}} .
$$

The coefficients $b_{i j}$ are bounded (it follows from (3.15) and (3.16) with $|\alpha|=0$ ).

Hence by (3.2),

$$
\left|\sum_{i, j=1}^{2} b_{i j}\left(Q_{r}^{*}\right) \frac{\partial \hat{\omega}\left(Q_{r}^{*}\right)}{\partial \xi_{i}} \frac{\partial \hat{v}\left(Q_{r}^{*}\right)}{\partial \xi_{j}}\right| \leqslant C\left[\left(\frac{\partial \hat{\omega}\left(Q_{r}^{*}\right)}{\partial \xi_{1}}\right)^{2}+\left(\frac{\partial \hat{\omega}\left(Q_{r}^{*}\right)}{\partial \xi_{2}}\right)^{2}\right]^{1 / 2}|\hat{v}|_{1, C_{2}}
$$

and

$$
\left|a_{h}^{*}(\omega, v)\right| \leqslant C \sum_{e}|\hat{v}|_{1, c_{2}} \sum_{r=1}^{4}\left[\left(\frac{\partial \hat{\omega}\left(Q_{r}^{*}\right)}{\partial \xi_{1}}\right)^{2}+\left(\frac{\partial \hat{\omega}\left(Q_{r}^{*}\right)}{\partial \xi_{2}}\right)^{2}\right]^{1 / 2} .
$$

We estimate the functional $L(\hat{u})=\partial \hat{\omega}\left(Q_{r}^{*}\right) / \partial \xi_{1}$ where $\hat{\omega}=\hat{u}-\hat{u}_{I}$. It is bounded on $H^{4}\left(C_{2}\right)$, it vanishes for $\hat{u} \in \hat{P}$ because $\hat{u}_{I}=\hat{u}$ in this case. If $\hat{u}=\xi_{2}^{3}$, then $\hat{u}_{I}=\xi_{2}$ and $\partial \hat{\omega} / \partial \xi_{1}=0$. If $\hat{u}=\xi_{1}^{3}$, then $\hat{u}_{I}=\xi_{1}$ and $\partial \hat{\omega}\left(Q_{r}^{*}\right) / \partial \xi_{1}=\left(3 \xi_{1}^{2}-1\right)_{\xi_{1}= \pm \sqrt{3} / 3}=0$. Hence $L(\hat{u})$ vanishes for $\hat{u} \in \hat{P}(3)$ and, according to the Bramble-Hilbert lemma, $\left|\partial \hat{\omega}\left(Q_{r}^{*}\right) / \partial \xi_{1}\right| \leqslant C|\hat{u}|_{4, C_{2}}$; in the same way we get $\left|\partial \hat{\omega}\left(Q_{r}^{*}\right) / \partial \xi_{2}\right| \leqslant C|\hat{u}|_{4, C_{2}}$. From (4.16), (2.9) and (3.3) we obtain

$$
\left|a_{h}^{*}\left(u-u_{I}, v\right)\right| \leqslant C \sum_{e}|\hat{u}|_{4, C_{2}}|\hat{v}|_{1, C_{2}} \leqslant C \sum_{e} h_{e}^{3}\|u\|_{4, e}|v|_{1, e} \leqslant C h^{3}\|u\|_{4, \Omega_{h}}|v|_{1, \Omega_{h}}
$$

The proof of (4.13) is similar.

(c) To prove (4.9) express $a(u, v)$ as follows:

$$
a(u, v)=\sum_{e} \int_{C_{2}} \sum_{i, j=1}^{2} J_{e} \hat{a}_{i j} \frac{\widehat{\partial u}}{\partial x_{i}} \frac{\widehat{\partial v}}{\partial x_{j}} d \xi
$$

With respect to definition of the error functional $E^{*}(\varphi)$ we get

$$
R_{h}^{*}(u, v)=\sum_{e} E^{*}\left(\sum_{i, j=1}^{2} J_{e} \hat{a}_{i j} \frac{\widehat{\partial u}}{\partial x_{i}} \frac{\widehat{\partial v}}{\partial x_{j}}\right) .
$$

We estimate $E^{*}\left(J_{e} \hat{a}_{11}\left(\widehat{\partial u} / \partial x_{1}\right)\left(\widehat{\partial v} / \partial x_{1}\right)\right)$. We have 


$$
J_{e} \frac{\partial \hat{v}}{\partial x_{1}}=J_{e}\left[\frac{\partial \hat{v}}{\partial \xi_{1}} \frac{\partial \xi_{1}^{e}}{\partial x_{1}}+\frac{\partial \hat{v}}{\partial \xi_{2}} \frac{\partial \xi_{2}^{e}}{\partial x_{1}}\right]=\frac{\partial x_{2}^{e}}{\partial \xi_{2}} \frac{\partial \hat{v}}{\partial \xi_{1}}-\frac{\partial x_{2}^{e}}{\partial \xi_{1}} \frac{\partial \hat{v}}{\partial \xi_{2}}
$$

so that

$$
E^{*}\left(J_{e} \hat{a}_{11} \frac{\partial u}{\partial x_{1}} \frac{\partial v}{\partial x_{1}}\right)=E^{*}\left(\frac{\partial x_{2}^{e}}{\partial \xi_{2}} \hat{a}_{11} \frac{\partial u}{\partial x_{1}} \frac{\partial \hat{v}}{\partial \xi_{1}}\right)-E^{*}\left(\frac{\partial x_{2}^{e}}{\partial \xi_{1}} \hat{a}_{11} \frac{\partial u}{\partial x_{1}} \frac{\partial \hat{v}}{\partial \xi_{2}}\right)
$$

To estimate the first term in (4.19) consider the functional $L(\sigma)=E^{*}\left(\sigma \partial \hat{v} / \partial \xi_{1}\right)-$ $H(\sigma) \forall \sigma \in H^{3}\left(C_{2}\right)$ where

$$
\begin{aligned}
H(\sigma)=\frac{1}{45} & \left\{\int_{-1}^{1} \frac{\partial^{2} \sigma\left(1, \xi_{2}\right)}{\partial \xi_{2}^{2}} \frac{\partial^{2} \hat{v}\left(1, \xi_{2}\right)}{\partial \xi_{2}^{2}} d \xi_{2}\right. \\
& \left.-\int_{-1}^{1} \frac{\partial^{2} \sigma\left(-1, \xi_{2}\right)}{\partial \xi_{2}^{2}} \frac{\partial^{2} \hat{v}\left(-1, \xi_{2}\right)}{\partial \xi_{2}^{2}} d \xi_{2}\right\} .
\end{aligned}
$$

If $\sigma=1, \xi_{1}, \xi_{2}, \xi_{1}^{2}, \xi_{1} \xi_{2}$, then from $\hat{v} \in \hat{P}$ (i.e., $\hat{v}$ is of the form (2.5)) and from (3.7) (which is satisfied by $E^{*}(\varphi)$ ) it follows $E^{*}\left(\sigma \partial \hat{v} / \partial \xi_{1}\right)=0$. Also $H(\sigma)=0$, hence $L(\sigma)$ $=0$. If $\sigma=\xi_{2}^{2}$, then an easy calculation gives $E^{*}\left(\sigma \partial \hat{v} / \partial \xi_{1}\right)=(16 / 45) \alpha_{8}=H(\sigma)\left(\alpha_{8}\right.$ is the last coefficient in (2.5)), thus $L(\sigma)=0$ for $\sigma \in \hat{P}(2)$. Further, from the explicit form of $L(\sigma)$,

$$
L(\sigma)=\int_{C_{2}} \sigma \frac{\partial \hat{v}}{\partial \xi_{1}} d \xi-\sum_{r=1}^{4} \sigma\left(Q_{r}^{*}\right) \frac{\partial \hat{v}\left(Q_{r}^{*}\right)}{\partial \xi_{1}}-H(\sigma),
$$

it follows by (3.2), the Sobolev lemma, the inequality $\int_{\partial C_{2}} \varphi^{2} d s \leqslant C\|\varphi\|_{1, C_{2}}^{2} \forall \varphi \in$ $H^{1}\left(C_{2}\right)$ and by (3.1) that $|L(\sigma)| \leqslant C|\hat{v}|_{1, C_{2}}\|\sigma\|_{3, C_{2}}$. Hence, the Bramble-Hilbert lemma gives

$$
|L(\sigma)| \leqslant C|\sigma|_{3, C_{2}}|\hat{v}|_{1, C_{2}}
$$

Therefore,

$\left|\sum_{e} E^{*}\left(\frac{\partial x_{2}^{e}}{\partial \xi_{2}} \hat{a}_{11} \frac{\partial \hat{u}}{\partial x_{1}} \frac{\partial \hat{v}}{\partial \xi_{1}}\right)\right| \leqslant C \sum_{e}|\sigma|_{3, C_{2}}|\hat{v}|_{1, C_{2}}+\left|\sum_{e} H(\sigma)\right|, \quad \sigma=\frac{\partial x_{2}^{e}}{\partial \xi_{2}} \hat{a}_{11} \frac{\widehat{\partial u}}{\partial x_{1}}$.

However, the last sum in the above inequality is equal to zero. In this sum they appear either integrals over element sides which lie on $\Gamma_{h}$; and, as $\left.v\right|_{\Gamma_{h}}=0$, we have $\partial^{2} \hat{v}\left( \pm 1, \xi_{2}\right) / \partial \xi_{2}^{2}=0$. Or they appear couples of integrals over a common side of two adjacent elements taken in opposite directions with integrands which are the same. The functions $a_{11} \partial u / \partial x_{1}$ as well as $v$ assume namely the same values on such side (they are continuous on $\bar{\Omega}_{h}$ ); also $x_{2}^{e}$ assume the same values on such side because these are quadratic polynomials in one variable determined uniquely by values at the three nodes of that side. So $\left(\partial^{2} \sigma / \partial \xi_{2}^{2}\right)\left(\partial^{2} \hat{v} / \partial \xi_{2}^{2}\right)$ assume the same values on such side. We have come to the bound

$$
\left|\sum_{e} E^{*}\left(\frac{\partial x_{2}^{e}}{\partial \xi_{2}} \hat{a}_{11} \frac{\partial u}{\partial x_{1}} \frac{\partial \hat{v}}{\partial \xi_{1}}\right)\right| \leqslant C \sum_{e}|\sigma|_{3, C_{2}}|\hat{v}|_{1, C_{2}}, \quad \sigma=\frac{\partial x_{2}^{e}}{\partial \xi_{2}} \hat{a}_{11} \frac{\partial u}{\partial x_{1}} .
$$


The other term in (4.19) can be estimated in the same way. In this case we set

$$
H(\sigma)=\frac{1}{45}\left\{\int_{-1}^{1} \frac{\partial^{2} \sigma\left(\xi_{1}, 1\right)}{\partial \xi_{1}^{2}} \frac{\partial^{2} \hat{v}\left(\xi_{1}, 1\right)}{\partial \xi_{1}^{2}} d \xi_{1}-\int_{-1}^{1} \frac{\partial^{2} \sigma\left(\xi_{1},-1\right)}{\partial \xi_{1}^{2}} \frac{\partial^{2} \hat{v}\left(\xi_{1},-1\right)}{\partial \xi_{1}^{2}} d \xi_{1}\right\}
$$

We find out that for $\sigma=1, \xi_{1}, \xi_{2}, \xi_{1} \xi_{2}, \xi_{2}^{2}$ both $E^{*}\left(\sigma \partial \hat{v} / \partial \xi_{2}\right)$ and $H(\sigma)$ vanish. For $\sigma=$ $\xi_{1}^{2}$ we get $E^{*}\left(\sigma \partial \hat{v} / \partial \xi_{2}\right)=H(\sigma)=(16 / 45) \alpha_{7}$. The remaining arguments are the same and lead again to the bound (4.22) where now $\sigma=\left(\partial x_{2}^{e} / \partial \xi_{1}\right) \hat{a}_{11} \widehat{\partial u} / \partial x_{1}$.

We must estimate $|\sigma|_{3, c_{2}}$. Take $\sigma=\left(\partial x_{2}^{e} / \partial \xi_{2}\right) \grave{a}_{11} \widehat{\partial u} / \partial x_{1}$ and express $\widehat{\partial u} / \partial x_{1}$ by rule of differentiation of composite functions. We find out that $\sigma$ is a linear combination of $\hat{a}_{11} \partial \hat{u} / \partial \xi_{1}$ and $\hat{a}_{11} \partial \hat{u} / \partial \xi_{2}$ with coefficients whose generic notation $\beta$ was introduced before and which satisfy (3.16). So set $\sigma=\beta \hat{a}_{11} \partial \hat{u} / \partial \xi_{i}$. As $u \in H^{4}(\Omega)$ and the mapping (2.7) satisfies (2.8), we have by Sobolev's lemma (applied to $\Omega^{0} \supset \bar{\Omega}_{h}$ ) $\max _{C_{2}}\left|D^{\alpha} \hat{u}\right| \leqslant C h_{e}^{|\alpha|}\|u\|_{4, \Omega}$ for $|\alpha| \leqslant 2$. Further, from $a_{11} \in H^{3}(\Omega)$ it follows $a_{11} \in$ $C^{1}\left(\bar{\Omega}_{h}\right)$ and $\max _{C_{2}}\left|D^{\alpha}\left(\beta \hat{a}_{11}\right)\right| \leqslant C h_{e}^{|\alpha|}$ for $|\alpha| \leqslant 1$ ( $\beta$ satisfies (3.16)). Therefore, (using (3.16) and (3.3))

$$
\begin{aligned}
\left|\beta \hat{a}_{11} \frac{\partial \hat{u}}{\partial \xi_{i}}\right|_{3, C_{2}} \leqslant C\left\{h_{e}\left|\beta \hat{a}_{11}\right|_{3, C_{2}}+h_{e}^{2}\left|\beta \hat{a}_{11}\right|_{2, C_{2}}\right\}\|u\|_{4, \Omega_{h}}+C\left\{h_{e}|\hat{u}|_{3, C_{2}}+|\hat{u}|_{4, C_{2}}\right\} \\
\leqslant C\left\{h_{e}^{4}\left\|\hat{a}_{11}\right\|_{0, C_{2}}+h_{e}^{3}\left|\hat{a}_{11}\right|_{1, C_{2}}+h_{e}^{2}\left|\hat{a}_{11}\right|_{2, C_{2}}+h_{e}\left|\hat{a}_{11}\right|_{3, C_{2}}\right\}\|u\|_{4, \Omega_{h}} \\
\quad+C\left\{h_{e}|\hat{u}|_{3, C_{2}}+|\hat{u}|_{4, C_{2}}\right\} \\
\leqslant C h_{e}^{3}\left\{\left\|a_{11}\right\|_{3, e}\|u\|_{4, \Omega_{h}}+\|u\|_{4, e}\right\} .
\end{aligned}
$$

The same bound is true for $\sigma=\left(\partial x_{2}^{e} / \partial \xi_{1}\right) \hat{a}_{11} \widehat{\partial u} / \partial x_{1}$. Consequently,

$$
\begin{aligned}
\left|\sum_{e} E^{*}\left(J_{e} \hat{a}_{11} \frac{\widehat{\partial u}}{\partial x_{1}} \frac{\widehat{\partial v}}{\partial x_{1}}\right)\right| & \leqslant C \sum_{e} h_{e}^{3}\left\{\left\|a_{11}\right\|_{3, e}\|u\|_{4, \Omega_{h}}+\|u\|_{4, e}\right\}|v|_{1, e} \\
& \leqslant C h^{3}\|u\|_{4, \Omega_{h}}|v|_{1, \Omega_{h}} .
\end{aligned}
$$

In the same way we can estimate the other terms in the first sum of (4.18). Thus, (4.9) is proved (see a remark following the equation (2.15)).

(d) We prove (4.10) for any formula with properties introduced in Lemma 3.6.

Let us first observe that estimating each term of the functional $E(\sigma \hat{v})$ (we use (3.2) and Sobolev's lemma) we obtain $|E(\sigma \hat{v})| \leqslant C\|\hat{v}\|_{0, C_{2}}\|\sigma\|_{2, C_{2}}$. If $\sigma \in \hat{P}(1)$, then by (3.7) or (3.8) and by (2.6) $E(\sigma \tilde{v})=0$. Therefore, by the Bramble-Hilbert lemma

$$
|E(\sigma \hat{v})| \leqslant C|\sigma|_{2, C_{2}}\|\hat{v}\|_{0, C_{2}} \quad \forall \sigma \in H^{2}\left(C_{2}\right), \forall \hat{v} \in \hat{P} .
$$

For $\sigma \in H^{3}\left(C_{2}\right)$ we get a better estimate. We have $E(\sigma \hat{v})=E\left(\left[\sigma-\sigma_{I}\right] \hat{v}\right)+$ $E\left(\sigma_{I} \hat{v}\right)$. By (4.23) and (3.6) the bound of the first term is

$$
\left|E\left(\left[\sigma-\sigma_{I}\right] \hat{v}\right)\right| \leqslant C\left|\sigma-\sigma_{I}\right|_{2, C_{2}}\|\hat{v}\|_{0, C_{2}} \leqslant C|\sigma|_{3, C_{2}}\|\hat{v}\|_{0, C_{2}} .
$$

Further, by (3.7) or (3.8) and by (2.6), (3.1)

$$
\left|E\left(\sigma_{I} \hat{v}\right)\right| \leqslant C\left\{\left|\sigma_{I}\right|_{2, C_{2}}|\hat{v}|_{3, C_{2}}+\left|\sigma_{I}\right|_{3, C_{2}}|\hat{v}|_{2, C_{2}}\right\} \quad \text { or } \quad\left|E\left(\sigma_{I} \hat{v}\right)\right| \leqslant C\left|\sigma_{I}\right|_{2, C_{2}}|\hat{v}|_{2, C_{2}},
$$


thus in both cases

$$
\left|E\left(\sigma_{I} \hat{v}\right)\right| \leqslant C\left\{\left|\sigma-\sigma_{I}\right|_{2, C_{2}}+|\sigma|_{2, C_{2}}\right\}|\hat{v}|_{1, C_{2}} \leqslant C|\sigma|_{2, C_{2}}|\hat{v}|_{1, C_{2}} .
$$

Hence,

$$
|E(\sigma \hat{v})| \leqslant C|\sigma|_{2, C_{2}}|\hat{v}|_{1, C_{2}}+C|\sigma|_{3, C_{2}}\|\hat{v}\|_{0, C_{2}} \quad \forall \sigma \in H^{3}\left(C_{2}\right), \forall \hat{v} \in \hat{P} .
$$

Now we must come back to the original notation: $\tilde{f}$ is the extension of $f$ defined on $R^{2}$ by $\tilde{f}=-\Sigma_{i, j=1}^{2} \partial\left(\tilde{a}_{i j} \partial \tilde{u} / \partial x_{j}\right) / \partial x_{i}$. We express $S_{h}(v)$ as follows:

$$
S_{h}(v)=\sum_{e}^{\prime} E\left(J_{e} \hat{\tilde{f}} \hat{v}\right)+\sum_{e}^{\prime \prime} E\left(J_{e} \hat{f} \hat{v}\right) .
$$

In the first sum the summation is taken over the boundary elements, in the second one over the inner elements (if $h$ is sufficiently small, the inner elements belong to $\Omega$; hence we may write $\hat{f}$ in the second sum).

Let us use (4.23) with $\sigma=J_{e} \hat{\tilde{f} \text {. }}$ As $D^{\alpha} J_{e}=O\left(h_{e}^{|\alpha|+2}\right.$ ) (it follows from (2.8)), we easily get

$$
\mid J_{e} \hat{\tilde{f}}_{2, C_{2}} \leqslant C\left\{h_{e}^{2}|\hat{\tilde{f}}|_{2, C_{2}}+h_{e}^{3}|\hat{\tilde{f}}|_{1, C_{2}}+h_{e}^{4}\|\hat{\tilde{f}}\|_{0, C_{2}}\right\} \leqslant C h_{e}^{3}\|\tilde{f}\|_{2, e}
$$

and

$$
\left|\sum_{e}^{\prime} E\left(J_{e} \hat{\tilde{f} \hat{v}}\right)\right| \leqslant C \sum_{e}^{\prime} h_{e}^{3}\|\tilde{f}\|_{2, e}\|\hat{v}\|_{0, C_{2}} .
$$

For the boundary elements Friedrichs' inequality gives $\|\hat{v}\|_{0, C_{2}} \leqslant C|\hat{v}|_{1, C_{2}}(\hat{v}$ vanishes on one side of $C_{2}$ ). Therefore

$$
\begin{aligned}
\left|\sum_{e}^{\prime} E\left(J_{e} \hat{\tilde{f} \hat{v}}\right)\right| & \leqslant C \sum_{e}^{\prime} h_{e}^{3}\|\tilde{f}\|_{2, e}|v|_{1, e} \leqslant C h^{3}\|\tilde{f}\|_{2, \Omega_{h}}|v|_{1, \Omega_{h}} \\
& \leqslant C h^{3}\|\tilde{u}\|_{4, \Omega_{h}}|v|_{1, \Omega_{h}} \leqslant C h^{3}\|u\|_{4, \Omega}|v|_{1, \Omega_{h}} .
\end{aligned}
$$

For the inner elements we use (4.24) with $\sigma=J_{e} \hat{f}$, and we easily get

Thus,

$$
\left|\sum_{e}^{\prime \prime} E\left(J_{e} \hat{f} \hat{v}\right)\right| \leqslant C h^{3}\|f\|_{3, \Omega}|v|_{1, \Omega_{h}} .
$$

$$
\begin{aligned}
\left|S_{h}(v)\right| & \leqslant C h^{3}\left[\|u\|_{4, \Omega_{h}}+\|f\|_{3, \Omega}\right]|v|_{1, \Omega_{h}} \quad \forall v \in V_{h}, \\
S_{h}(v) & =(f, v)_{0, \Omega_{h}}-f_{h}(v) .
\end{aligned}
$$

Remark 5. Relaxing one assumption of the theorem, changing namely the condition of 2 -strong regularity into 1 -strong regularity, one can prove in the same way that

$$
\left\|u-u_{h}^{*}\right\|_{1, \Omega \cap \Omega_{h}} \leqslant C h^{2} .
$$

(2.8) is satisfied for $|\alpha| \leqslant 2$ if instead of (2.11) we require $\rho\left(a_{i}, a_{i}^{\prime}\right) \leqslant C_{2} h_{e}^{2}$ (see Remark 2).

Theorem 4.2 introduced below shows that the superconvergence phenomenon is not closely connected with Gauss' $2 \times 2$ formula. For Theorem 4.2 we need that the finite element partitions, besides being 2 -strongly regular, are such that 


$$
\left|J_{e}^{-1} \frac{\partial x_{i}^{e}}{\partial \xi_{1}} \frac{\partial x_{j}^{e}}{\partial \xi_{2}}-J_{e^{1}}^{-1} \frac{\partial x_{i}^{e^{1}}}{\partial \xi_{1}} \frac{\partial x_{j}^{e^{1}}}{\partial \xi_{2}}\right| \leqslant C h, \quad i, j=1,2
$$

for any two adjacent elements $e, e^{1}$.

Remark 6. Condition (4.26) is of different nature than conditions (2.8) and (2.9) because it does not concern single elements. If (4.26) is satisfied and the coefficients $a_{i j}$ are Lipschitz continuous, then the difference of values of the coefficient $b_{12}$ (see (3.15)) on adjacent elements is $O(h)$. We give a sufficient condition that (4.26) be fulfilled. It is similar to conditions given in Remark 2.

We ask again (2.10) and instead of (2.11) a weaker condition

$$
\rho\left(a_{i}, a_{i}^{\prime}\right) \leqslant C_{2} h_{e}^{2}, \quad 1 \leqslant i \leqslant 8 .
$$

However, we add a third condition. Let us denote by $\alpha_{e}$ and $\beta_{e}$ the angles which make the sides $a_{1}^{\prime} a_{2}^{\prime}$ and $a_{1}^{\prime} a_{4}^{\prime}$, respectively, with the $x_{1}$-axis (if a suitable notation is used then $\left.\left|\beta_{e}-\alpha_{e}\right|=\omega_{e}\right)$. The condition reads

$$
\left|\alpha_{e}-\alpha_{e^{1}}\right| \leqslant C_{3} h, \quad\left|\beta_{e}-\beta_{e^{1}}\right| \leqslant C_{3} h
$$

for any two adjacent elements $e, e^{1}$.

The proof that this condition is sufficient is simple: For the parallelogram $e^{\prime}$ corresponding to the element $e$ one computes

$$
\frac{\partial x_{1}^{e^{\prime}}}{\partial \xi_{1}}=\frac{1}{2} h_{e} \cos \alpha_{e}, \frac{\partial x_{1}^{e^{\prime}}}{\partial \xi_{2}}=\frac{1}{2} k_{e} \cos \beta_{e}, \frac{\partial x_{2}^{e^{\prime}}}{\partial \xi_{1}}=\frac{1}{2} h_{e} \sin \alpha_{e}, \frac{\partial x_{2}^{e^{\prime}}}{\partial \xi_{2}}=\frac{1}{2} k_{e} \sin \beta_{e} .
$$

Hence, $J_{e^{\prime}}=1 / 4 h_{e} k_{e} \sin \omega_{e}$ and, for instance,

$$
J_{e^{-1}} \frac{\partial x_{1}^{e^{\prime}}}{\partial \xi_{1}} \frac{\partial x_{1}^{e^{\prime}}}{\partial \xi_{2}}=\frac{\cos \alpha_{e} \cos \beta_{e}}{\sin \omega_{e}}
$$

From (4.27) and $k_{e} / h_{e} \geqslant C_{3}$ we easily get

$$
J_{e}^{-1} \frac{\partial x_{1}^{e}}{\partial \xi_{1}} \frac{\partial x_{1}^{e}}{\partial \xi_{2}}=\frac{\cos \alpha_{e} \cos \beta_{e}}{\sin \omega_{e}}+O\left(h_{e}\right)
$$

From (2.10) and (4.28) it follows (4.26) for $i=j=1$.

THEOREM 4.2. Let the quadrature formula (2.16) be either a symmetric formula with positive coefficients which integrates exactly all polynomials from $\hat{Q}(3)$ or a formula which integrates exactly all polynomials from $\hat{P}(4)$. Let the finite element partitions, besides being 2-strongly regular, satisfy (4.26), and let the remaining assumptions of Theorem 4.1 be fulfilled. Then there exists a constant $C$ independent of $h$ (it is of the form $C_{1}\|u\|_{4, \Omega}+C_{2}\|f\|_{3, \Omega}$ ) such that

$$
\left\|u-u_{h}\right\|_{h} \leqslant C h^{3} \text {. }
$$

Proof. (a) Distinguish two cases: $I(\varphi)$ is of the form (3.10) where $I^{0}(\varphi)$ integrates exactly all polynomials from $\hat{P}(4)$ or $I(\varphi)$ itself has this property. The other possibility is included in the preceding one because $I(\varphi)$ is again of the form (3.10) with $\alpha=0$. The second case is that $I(\varphi)$ is of the form (3.11) where $E^{1}(\varphi)$ satisfies (3.8). Consider the first case. Then 


$$
a_{h}(u, v)=(f, v)_{0, \Omega_{h}}-\alpha R_{h}^{*}(u, v)-(1-\alpha) R_{h}^{0}(u, v) \quad \forall v \in V_{h},
$$

$$
R_{h}^{0}(w, v)=a(w, v)-a_{h}^{0}(w, v)
$$

and $a_{h}^{0}(w, v)$ is the approximate value of $a(w, v)$ computed by means of the formula $I^{0}(\varphi)$ (or $I(\varphi)$ if $\left.\alpha=0\right)$. Hence, subtracting (2.19), we get

$$
a_{h}\left(u-u_{h}, v\right)=S_{h}(v)-\alpha R_{h}^{*}(u, v)-(1-\alpha) R_{h}^{0}(u, v) \quad \forall v \in V_{h},
$$

where $S_{h}(v)$ is defined in (4.25). Adding $a_{h}\left(u_{I}-u, v\right)$ to both sides, we easily obtain

$$
\begin{aligned}
a_{h}\left(u_{I}-u_{h}, v\right)= & S_{h}(v)-\alpha R_{h}^{*}(u, v)+\alpha a_{h}^{*}\left(u_{I}-u, v\right)-(1-\alpha) R_{h}^{0}\left(u_{I}, v\right) \\
& +(1-\alpha) a\left(u_{I}-u, v\right) \quad \forall v \in V_{h} .
\end{aligned}
$$

Suppose that we prove

$$
\begin{gathered}
\left|R_{h}^{0}\left(u_{I}, v\right)\right| \leqslant C h^{3}|v|_{1, \Omega_{h}} \quad \forall v \in V_{h}, \\
\left|a\left(u-u_{I}, v\right)\right| \leqslant C h^{3}|v|_{1, \Omega_{h}} \quad \forall v \in V_{h} .
\end{gathered}
$$

Then putting $v=u_{I}-u_{h}$ in (4.31) we get, by (4.25), (4.9), (4.8), (4.32), (4.33) and (3.9)

$$
\left|u_{I}-u_{h}\right|_{1, \Omega_{h}} \leqslant C h^{3} \text {. }
$$

Consequently, by (4.15), (4.13), (4.34) and (4.2)

$$
\left\|u-u_{h}\right\|_{h} \leqslant\left\|u-u_{I}\right\|_{h}+\left\|u_{I}-u_{h}\right\|_{h} \leqslant C h^{3}+c_{4}\left|u_{I}-u_{h}\right|_{1, \Omega_{h}} \leqslant C h^{3} .
$$

(b) To prove (4.32) we express $a\left(u_{I}, v\right)$ as follows:

$$
a\left(u_{I}, v\right)=\sum_{e} \int_{C_{2}} \sum_{i, j=1}^{2} b_{i j} \frac{\partial \hat{u}_{I}}{\partial \xi_{i}} \frac{\partial \hat{v}}{\partial \xi_{j}} d \xi
$$

$\left(b_{i j}\right.$ are the coefficients (3.15)). Hence,

$$
R_{h}^{0}\left(u_{I}, v\right)=\sum_{e} E^{0}\left(\sum_{i, j=1}^{2} b_{i j} \frac{\partial \hat{u}_{I}}{\partial \xi_{i}} \frac{\partial \hat{v}}{\partial \xi_{j}}\right)
$$

here $E^{0}(\varphi)$ is the error functional associated to $I^{0}(\varphi)$ and satisfying (3.8). The coefficients $b_{i j}$ are linear combinations of terms of the form $\beta \hat{a}_{m n}$, where $\beta$ satisfies (3.16). So it is sufficient to prove

$$
\left|E^{0}\left(\beta \hat{a}_{m n} \frac{\partial \hat{u}_{I}}{\partial \xi_{i}} \frac{\partial \hat{v}}{\partial \xi_{j}}\right)\right| \leqslant C h_{e}^{3}\|u\|_{3, e}|v|_{1, e},
$$

and (4.32) follows immediately.

Set $\sigma=\beta \hat{a}_{m n} \partial \hat{u}_{I} / \partial \xi_{i}$. We have

$$
E^{0}\left(\sigma \frac{\partial \hat{v}}{\partial \xi_{j}}\right)=E^{0}\left(\left[\sigma-\sigma_{I}\right] \frac{\partial \hat{v}}{\partial \xi_{j}}\right)+E^{0}\left(\sigma_{I} \frac{\partial \hat{v}}{\partial \xi_{j}}\right) .
$$

We estimate the first term by means of (4.23): 


$$
\left|E^{0}\left(\left[\sigma-\sigma_{I}\right] \frac{\partial \hat{v}}{\partial \xi_{j}}\right)\right| \leqslant C\left|\sigma-\sigma_{I}\right|_{2, C_{2}}|\hat{v}|_{1, C_{2}} \leqslant C|\sigma|_{3, C_{2}}|\hat{v}|_{1, C_{2}} .
$$

Now we use (3.8) and get

$$
\begin{aligned}
\left|E^{0}\left(\sigma_{I} \frac{\partial \hat{v}}{\partial \xi_{j}}\right)\right| & \leqslant C\left|\sigma_{I}\right|_{3, C_{2}}|\hat{v}|_{3, C_{2}} \leqslant C\left\{\left|\sigma-\sigma_{I}\right|_{3, C_{2}}+|\sigma|_{3, C_{2}}\right\}|\hat{v}|_{1, C_{2}} \\
& \leqslant C|\sigma|_{3, C_{2}}|\hat{v}|_{1, C_{2}} .
\end{aligned}
$$

Thus $\left|E^{0}\left(\sigma \partial \hat{v} / \partial \xi_{j}\right)\right| \leqslant C|\sigma|_{3, C_{2}}|\hat{v}|_{1, C_{2}}$. Further, from (3.16), (3.2) and $a_{m n} \in C^{1}\left(\bar{\Omega}_{h}\right)$ it follows

$$
\begin{aligned}
& \left|\beta \hat{a}_{m n} \frac{\partial \hat{u}_{I}}{\partial \xi_{i}}\right|_{3, C_{2}} \leqslant C\left\{\left|\beta \frac{\partial \hat{u}_{I}}{\partial \xi_{i}}\right|_{3, C_{2}}+h_{e}\left|\beta \frac{\partial \hat{u}_{I}}{\partial \xi_{i}}\right|_{2, C_{2}}+\left[h_{e}\left|\hat{u}_{I}\right|_{1, C_{2}}+\left|\hat{u}_{I}\right|_{2, C_{2}}\right]\left|\hat{a}_{m n}\right|_{2, C_{2}}\right. \\
& \left.+\left|\hat{u}_{I}\right|_{1, c_{2}}\left|\hat{a}_{m n}\right|_{3, c_{2}}\right\} \\
& \leqslant C\left\{h_{e}^{3}\left|\hat{u}_{I}\right|_{1, C_{2}}+h_{e}^{2}\left|\hat{u}_{I}\right|_{2, C_{2}}+h_{e}\left|\hat{u}_{I}\right|_{3, C_{2}}\right. \\
& \left.+h_{e}\left[h_{e}\left|\hat{u}_{I}\right|_{1, C_{2}}+\left|\hat{u}_{I}\right|_{2, C_{2}}\right]\left|a_{m n}\right|_{2, e}+h_{e}^{2}\left|\hat{u}_{I}\right|_{1, C_{2}}\left|a_{m n}\right|_{3, e}\right\} \text {. }
\end{aligned}
$$

We use the bound

$$
\left|\hat{u}_{I}\right|_{j, C_{2}} \leqslant\left|\hat{u}_{I}-\hat{u}\right|_{j, C_{2}}+|\hat{u}|_{j, C_{2}} \leqslant C\left\{|\hat{u}|_{3, C_{2}}+|\hat{u}|_{j, C_{2}}\right\}
$$

$j=1,2,3$, and the fact that, due to (4.3), $u \in C^{2}\left(\bar{\Omega}_{h}\right)$, and we get the final bound

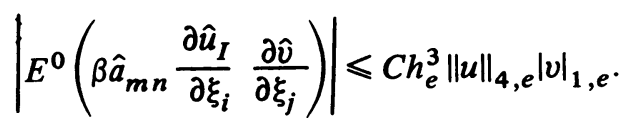

(c) The main problem is to prove (4.33). We have

$$
a(\omega, v)=\sum_{e} \int_{C_{2}} \sum_{i, j=1}^{2} b_{i j} \frac{\partial \hat{\omega}}{\partial \xi_{i}} \frac{\partial \hat{v}}{\partial \xi_{j}} d \xi
$$

(again $\omega=u-u_{I}$ ). We must estimate the terms $\int_{C_{2}} b_{i j}\left(\partial \hat{\omega} / \partial \xi_{i}\right)\left(\partial \hat{v} / \partial \xi_{j}\right) d \xi$. We may restrict ourselves to two cases: $i=j=1, i=1, j=2$. Consider first $\int_{C_{2}} b_{11}\left(\partial \hat{\omega} / \partial \xi_{1}\right) \cdot$ $\left(\partial \hat{v} / \partial \xi_{1}\right) d \xi$. From (3.16) and $a_{i j} \in C^{1}\left(\bar{\Omega}_{h}\right)$ it follows $b_{i j}=b_{i j}^{0}+O\left(h_{e}\right)$, where $b_{i j}^{0}$ denotes again the value of $b_{i j}$ at the center. Hence

$$
\int_{C_{2}} b_{11} \frac{\partial \hat{\omega}}{\partial \xi_{1}} \frac{\partial \hat{v}}{\partial \xi_{1}} d \xi=b_{11}^{0} \int_{C_{2}} \frac{\partial \hat{\omega}}{\partial \xi_{1}} \frac{\partial \hat{v}}{\partial \xi_{1}} d \xi+\int_{C_{2}} O\left(h_{e}\right) \frac{\partial \hat{\omega}}{\partial \xi_{1}} \frac{\partial \hat{v}}{\partial \xi_{1}} d \xi .
$$

As $|\hat{\omega}|_{1, C_{2}} \leqslant C|\hat{u}|_{3, C_{2}}$, the second term is bounded by $C h_{e}|\hat{u}|_{3, C_{2}}|\hat{v}|_{1, C_{2}} \leqslant$ $C h_{e}^{3}\|u\|_{3, e}|v|_{1, e}$. To estimate the first term consider the functional $L(\hat{u})=$ $\int_{C_{2}}\left(\partial \hat{\omega} / \partial \xi_{1}\right)\left(\partial \hat{v} / \partial \xi_{1}\right) d \xi$. It vanishes for $\hat{u} \in \hat{P}$. If $\hat{u}=\xi_{2}^{3}$, then $\hat{\omega}=\xi_{2}^{3}-\xi_{2}, \partial \hat{\omega} / \partial \xi_{1}$ $=0$ and $L$ vanishes. If $\hat{u}=\xi_{1}^{3}$, then $\partial \hat{\omega} / \partial \xi_{1}=2\left((3 / 2) \xi_{1}^{2}-1 / 2\right)$, i.e. $\partial \hat{\omega} / \partial \xi_{1}$ is a multiple of the Legendre polynomial $P_{2}\left(\xi_{1}\right)$; and as $\partial \hat{v} / \partial \xi_{1}$ is a linear polynomial of $\xi_{1}$ and integration with respect to $\xi_{1}$ is done over the interval $\langle-1,1\rangle, L$ vanishes, too. The 
Bramble-Hilbert lemma implies $|L(\hat{u})| \leqslant C|\hat{u}|_{4, C_{2}}|\hat{v}|_{1, C_{2}} \leqslant C h_{e}^{3}\|u\|_{4, e}|v|_{1, e}$. Hence,

$$
\left|\sum_{e} \int_{C_{2}} \sum_{i=1}^{2} b_{i i} \frac{\partial \hat{\omega}}{\partial \xi_{i}} \frac{\partial \hat{v}}{\partial \xi_{i}} d \xi\right| \leqslant C \sum_{e} h_{e}^{3}\|u\|_{4, e}|v|_{1, e} \leqslant C h^{3}\|u\|_{4, \Omega_{h}}|v|_{1, \Omega_{h}} .
$$

Now let us consider the integral

$$
\int_{C_{2}} b_{12} \frac{\partial \hat{\omega}}{\partial \xi_{1}} \frac{\partial \hat{v}}{\partial \xi_{2}} d \xi=b_{12}^{0} \int_{C_{2}} \frac{\partial \hat{\omega}}{\partial \xi_{1}} \frac{\partial \hat{v}}{\partial \xi_{2}} d \xi+\int_{C_{2}} O\left(h_{e}\right) \frac{\partial \hat{\omega}}{\partial \xi_{1}} \frac{\partial \hat{v}}{\partial \xi_{2}} d \xi
$$

The second term and the sum of these terms are bounded as above. To estimate the first term we introduce the functional

$$
L(\hat{u})=\int_{C_{2}} \frac{\partial \hat{\omega}}{\partial \xi_{1}} \frac{\partial \hat{v}}{\partial \xi_{2}} d \xi-H(\hat{u})
$$

$$
H(\hat{u})=\frac{1}{15}\left\{\int_{-1}^{1} \frac{\partial^{2} \hat{\omega}\left(\xi_{1}, 1\right)}{\partial \xi_{1}^{2}} \frac{\partial \hat{v}\left(\xi_{1}, 1\right)}{\partial \xi_{1}} d \xi_{1}-\int_{-1}^{1} \frac{\partial^{2} \hat{\omega}\left(\xi_{1},-1\right)}{\partial \xi_{1}^{2}} \frac{\partial \hat{v}\left(\xi_{1},-1\right)}{\partial \xi_{1}} d \xi_{1}\right\} .
$$

If $\hat{u} \in \hat{P}$, then $\hat{\omega}=0$ and $L(\hat{u})=0$. If $\hat{u}=\xi_{2}^{3}$, then $\partial \hat{\omega} / \partial \xi_{1}=0$ and $L(\hat{u})=0$. If $\hat{u}=\xi_{1}^{3}$, then $\int_{C_{2}}\left(\partial \hat{\omega} / \partial \xi_{1}\right)\left(\partial \hat{v} / \partial \xi_{2}\right) d \xi=(16 / 15) \alpha_{7}=H(\hat{u})$ and $L(\hat{u})=0$. The Bramble-Hilbert lemma implies $|L(\hat{u})| \leqslant C|\hat{u}|_{4, C_{2}}|\hat{v}|_{1, C_{2}} \leqslant C h_{e}^{3}\|u\|_{4, e}|v|_{1, e}$. Thus,

$$
\begin{aligned}
\left|\sum_{e} b_{12}^{0} \int_{C_{2}} \frac{\partial \hat{\omega}}{\partial \xi_{1}} \frac{\partial \hat{v}}{\partial \xi_{2}} d \xi\right| & \leqslant C \sum_{e} h_{e}^{3}\|u\|_{4, e}|v|_{1, e}+\left|\sum_{e} b_{12}^{0} H(\hat{u})\right| \\
& \leqslant C h^{3}\|u\|_{4, \Omega_{h}}|v|_{1, \Omega_{h}}+\left|\sum_{e} b_{12}^{0} H(\hat{u})\right| .
\end{aligned}
$$

In the sum $\Sigma_{e} b_{12}^{0} H(\hat{u})$ they appear either integrals over element sides which lie on $\Gamma_{h}$; and because $\left.v\right|_{\Gamma_{h}}=0$, thus $\partial \hat{v}\left(\xi_{1}, \pm 1\right) / \partial \xi_{1}=0$, these integrals vanish. Or they appear couples of integrals over a common side of two adjacent elements taken in opposite directions with integrands which are the same ( $\omega$ and $v$ are continuous on $\bar{\Omega}_{h}$, hence they assume the same values on each side). The factors $b_{12}^{0}$ need not be the same; however, their difference is $O(h)$ according to Remark 6. Therefore, (we use again the inequality $\int_{\partial C_{2}} \varphi^{2} d s \leqslant C\|\varphi\|_{1, C_{2}}^{2}$ ) by (3.2), (3.1) and (3.6)

$$
\begin{gathered}
\left|\sum_{e} b_{12}^{0} H(\hat{u})\right| \leqslant C h \sum_{e}\left|\int_{-1}^{1} \frac{\partial^{2} \hat{\omega}\left(\xi_{1}, 1\right)}{\partial \xi_{1}^{2}} \frac{\partial \hat{v}\left(\xi_{1}, 1\right)}{\partial \xi_{1}} d \xi_{1}\right| \\
\leqslant \operatorname{Ch} \sum_{e}|| \frac{\partial^{2} \hat{\omega}}{\partial \xi_{1}^{2}} \frac{\partial \hat{v}}{\partial \xi_{1}}\left\|_{1, C_{2}} \leqslant C h \sum_{e}\right\| \hat{\omega} \|_{3, C_{2}}|\hat{v}|_{1, C_{2}} \leqslant C h \sum_{e}|\hat{u}|_{3, C_{2}}|\hat{v}|_{1, C_{2}} \\
\leqslant \operatorname{Ch} \sum_{e} h_{e}^{2}\|u\|_{3, e}|v|_{1, e} \leqslant C h^{3}\|u\|_{3, \Omega_{h}}|v|_{1, \Omega_{h}}
\end{gathered}
$$

and the proof of (4.33) is finished.

(d) Let us consider the second case of $I(\varphi)$, namely $I(\varphi)$ is of the form (3.11). Then $a_{h}(w, v)=a_{h}^{*}(w, v)+R_{h}^{1}(w, v)$, where 


$$
R_{h}^{1}(w, v)=\sum_{e} E^{1}\left(\sum_{i, j=1}^{2} b_{i j} \frac{\partial \hat{w}}{\partial \xi_{i}} \frac{\partial \hat{v}}{\partial \xi_{j}}\right)
$$

We have $a_{h}(u, v)=(f, v)_{0, \Omega_{h}}-a(u, v)+a_{h}(u, v)=(f, v)_{0, \Omega_{h}}-R_{h}^{*}(u, v)+R_{h}^{1}(u, v)$, and we easily get

$$
a_{h}\left(u_{I}-u_{h}, v\right)=S_{h}(v)-K_{h}^{*}(u, v)+R_{h}^{1}\left(u_{I}, v\right)+a_{h}^{*}\left(u_{I}-u, v\right) \quad \forall v \in V_{h}
$$

If we show that

$$
\left|R_{h}^{1}\left(u_{I}, v\right)\right| \leqslant C h^{3}|v|_{1, \Omega_{h}} \quad \forall v \in V_{h},
$$

then using the arguments of part (a) of the proof we come to (4.29). The proof of (4.39) is the same as that of (4.32) because $R_{h}^{1}\left(u_{I}, v\right)$ and $R_{h}^{0}\left(u_{I}, v\right)$ have the same form (see (4.35) and (4.37)) and $E^{1}(\varphi)$ has the same property as $E^{0}(\varphi)$, namely it satisfies (3.8).

5. Superconvergence in Three Dimensions. A three-dimensional isoparametric quadratic element of the Serpendipity family has 20 nodes corresponding to 20 nodes of the three-dimensional cube $C_{3}:-1 \leqslant \xi_{i} \leqslant 1, i=1,2,3$. The nodes of $C_{3}$ are vertices of $C_{3}$ and midpoints of sides. The space $\hat{P}$ consists of incomplete quartic polynomials of the form

$$
\begin{array}{r}
\alpha_{1}+\alpha_{2} \xi_{1}+\alpha_{3} \xi_{2}+\alpha_{4} \xi_{3}+\alpha_{5} \xi_{1}^{2}+\alpha_{6} \xi_{1} \xi_{2}+\alpha_{7} \xi_{1} \xi_{3}+\alpha_{8} \xi_{2}^{2}+\alpha_{9} \xi_{2} \xi_{3} \\
+\alpha_{10} \xi_{3}^{2}+\alpha_{11} \xi_{1}^{2} \xi_{2}+\alpha_{12} \xi_{1}^{2} \xi_{3}+\alpha_{13} \xi_{1} \xi_{2}^{2}+\alpha_{14} \xi_{1} \xi_{3}^{2}+\alpha_{15} \xi_{2}^{2} \xi_{3} \\
+\alpha_{16} \xi_{2} \xi_{3}^{2}+\alpha_{17} \xi_{1} \xi_{2} \xi_{3}+\alpha_{18} \xi_{1}^{2} \xi_{2} \xi_{3}+\alpha_{19} \xi_{1} \xi_{2}^{2} \xi_{3}+\alpha_{20} \xi_{1} \xi_{2} \xi_{3}^{2}
\end{array}
$$

$\hat{P}$ satisfies again (2.6). The functions $N_{j}\left(\xi_{1}, \xi_{2}, \xi_{3}\right)$ can be found in [8, p. 121]. The definition of a $k$-strongly regular partition is the same as in two dimensions with one exception: instead of (2.9) we have to require

$$
c_{2}^{-1} h_{e}^{3} \leqslant\left|J_{e}\right| \leqslant c_{2} h_{e}^{3} .
$$

With exception of these changes the definition of $V_{h}$ is the same as before. The definition of $u_{h}^{*}$ is $a_{h}^{*}\left(u_{h}^{*}, v\right)=f_{h}^{*}(v) \forall v \in V_{h}$, where

$$
\begin{aligned}
a_{h}^{*}(w, v) & =\sum_{e} \sum_{r=1}^{8} J_{e}\left(Q_{r}^{*}\right) \sum_{i, j=1}^{3} \hat{a}_{i j} \frac{\widehat{\partial w}}{\partial x_{i}}\left(Q_{r}^{*}\right) \frac{\widehat{\partial v}}{\partial x_{j}}\left(Q_{r}^{*}\right), \\
f_{h}^{*}(v) & =\sum_{e} \sum_{r=1}^{8} J_{e}\left(Q_{r}^{*}\right) \hat{f}\left(Q_{r}^{*}\right) \hat{v}\left(Q_{r}^{*}\right), \\
Q_{r}^{*} & =\left( \pm \frac{\sqrt{3}}{3}, \pm \frac{\sqrt{3}}{3}, \pm \frac{\sqrt{3}}{3}\right) .
\end{aligned}
$$

The same superconvergence theorem as in two dimensions is true with one change concerning the assumptions. We have to assume that the partitions are 3-strongly regular. The reason is the following: in two dimensions the derivatives $D^{\alpha} x_{i}^{e}$ of 2- 
strongly partitions are bounded by $h_{e}^{|\alpha|}$ not only for $|\alpha| \leqslant 3$ but for all $\alpha$ because $x_{i}^{e}$ are cubic polynomials. We need this fact to prove (3.16). In three dimensions $x_{i}^{e}$ are quartic polynomials, so we have to assume the 3-strong regularity. The proof of the theorem is similar to the proof of Theorem 4.1 , and we leave it out.

6. Numerical Results and Application of Superconvergence in Practical Computations. The following problem was solved:*

$-\Delta u=-2 y+54 x y-12 x y^{2}+16 y^{2}-14 y^{3}-4 x^{3}-12 x+16 x^{2}-42 x^{2} y$ in $\Omega$,

$$
\left.u\right|_{\Gamma}=0, \quad \Omega: 0<x<1,0<y<1 .
$$

The exact solution is $u(x, y)=x(1-x) y(1-y)(1+2 x+7 y)$. We used partitions consisting of square elements with vertices $\{(i h, j h)\}_{i, j=0}^{M}, M=h^{-1}, h=1 / 4,1 / 5,1 / 6$, $1 / 7,1 / 8$ and Gauss' $2 \times 2$ formula. The norm $\left\|u-u_{h}^{*}\right\|_{h}$ is denoted by $E_{G}$ and is equal in this case to

$$
E_{G}=\left\{N_{G}^{-1} \sum_{P \in G}\left[\left(\frac{\partial\left(u-u_{h}^{*}\right)(P)}{\partial x}\right)^{2}+\left(\frac{\partial\left(u-u_{h}^{*}\right)(P)}{\partial y}\right)^{2}\right]\right\}^{1 / 2} .
$$

Here $N_{G}=4 h^{-2}$ is the number of Gaussian points. Also, the gradient at vertices of square elements was computed (the unique values of the gradient were won by averaging); and, as a measure of the error, the number

$$
E_{V}=\left\{N_{V}^{-1} \sum_{P \in V}\left[\left(\frac{\partial\left(u-u_{h}^{*}\right)(P)}{\partial x}\right)^{2}+\left(\frac{\partial\left(u-u_{h}^{*}\right)(P)}{\partial y}\right)^{2}\right]\right\}^{1 / 2}
$$

is taken. The set $V$ consists of all vertices of square elements with exception of the vertices of $\Omega$. Table 1 shows on one hand the big difference between the magnitudes of $E_{G}$ and $E_{V}$ and the superconvergence at Gaussian points; on the other hand it shows that $E_{V}$ goes to zero just as fast as $h^{2}$, i.e. $h^{-2} E_{V} \rightarrow$ const $>0$.

TABLE 1

\begin{tabular}{lcccc}
\hline$h$ & $E_{G} \times 10^{3}$ & $E_{V} \times 10^{3}$ & $h^{-3} E_{G}$ & $h^{-2} E_{V}$ \\
\hline$\frac{1}{4}$ & 9.0 & 41 & 0.57 & 0.65 \\
\hline$\frac{1}{5}$ & 4.2 & 25 & 0.52 & 0.64 \\
\hline$\frac{1}{6}$ & 2.2 & 18 & 0.49 & 0.63 \\
\hline$\frac{1}{7}$ & 1.3 & 13 & 0.46 & 0.63 \\
\hline$\frac{1}{8}$ & 0.85 & 10 & 0.43 & 0.63 \\
\hline
\end{tabular}

\footnotetext{
* The author is indebted to M. Kovaríková who carried out all computations on the computer DATASAAB D21.
} 
Gauss" $3 \times 3$ formula and Čebyšev's product formula with nine points were also applied. The values $E_{G}$ and $E_{V}$ differ less than $0.2 \%$ from values given in Table 1 (let us emphasize that whatever formula is applied, the set $G$ is the set of maps of points $Q_{r}^{*}(r=1, \ldots, 4)$ of Gauss' $2 \times 2$ formula $)$.

The problem (6.1) was solved by curved elements not satisfying (2.8). The square elements were distorted into curved ones in that the midpoints of two sides of each element were moved in the $x$-direction and $y$-direction, respectively. The length of these displacements was always the same: $1 / 2 h^{2}$. Such elements do not satisfy (2.8) as $\partial^{3} x_{1}^{e} / \partial \xi_{1} \partial \xi_{2}^{2}$ and $\partial^{3} x_{2}^{e} / \partial \xi_{1}^{2} \partial \xi_{2}$ are in absolute value equal to $1 / 2 h^{2}$. The number $E_{G}$ is not equal to $\left\|u-u_{h}^{*}\right\|_{h}$; however, it is an equivalent norm; in addition $E_{G}^{-1}\left\|u-u_{h}^{*}\right\|_{h}$ $=1+O(h)$. Table 2 indicates convincingly that $E_{G} \geqslant c h^{2}, c>0$, i.e. there is no superconvergence. Nevertheless, $E_{G}$ is still substantially smaller than $E_{V}$.

TABLE 2

\begin{tabular}{lccc}
\hline$h$ & $E_{G} \times 10^{2}$ & $h^{-2} E_{G}$ & $E_{V} \times 10^{2}$ \\
\hline$\frac{1}{4}$ & 4.4 & 0.70 & 15 \\
\hline$\frac{1}{5}$ & 3.1 & 0.76 & 5.7 \\
\hline$\frac{1}{6}$ & 2.3 & 0.83 & 5.9 \\
\hline$\frac{1}{7}$ & 2.0 & 0.97 & 3.9 \\
\hline$\frac{1}{8}$ & 1.3 & 0.80 & 3.4 \\
\hline
\end{tabular}

Gauss' $3 \times 3$ formula gives values of $E_{G}$ which differ less than $4 \%$ from values given in Table 2.

In general, if we compute the gradient at Gaussian points we always can expect much more accurate values than at vertices. If a greater part of the elements differ little from parallelepipeds we reach even a greater improvement of accuracy. The question is what integration formula to choose. Theorems 4.1 and 5.1 and Remark 5 show that very often Gauss' $2 \times 2$ or $2 \times 2 \times 2$ formula can be sufficient. However, Gauss' $3 \times 3$ or $3 \times 3 \times 3$ formula guarantees that, both in case of superconvergence as well as in case that superconvergence does not set in (see [4, pp. 462-463]), we retain the highest order of accuracy which is possible.

The usual requirement of users of finite element codes is to get the values of gradient at vertices of elements. These values must be interpolated from values at Gaussian points. If there is no superconvergence, i.e. the rate of convergence in the $\|\cdot\|_{h}$-norm is $O\left(h^{2}\right)$ and not better, then interpolation from four Gaussian points on each element by a linear isoparametric shape function is sufficient. Evidently, such interpolation would make worse the accuracy won in case of superconvergence. Therefore, a better 


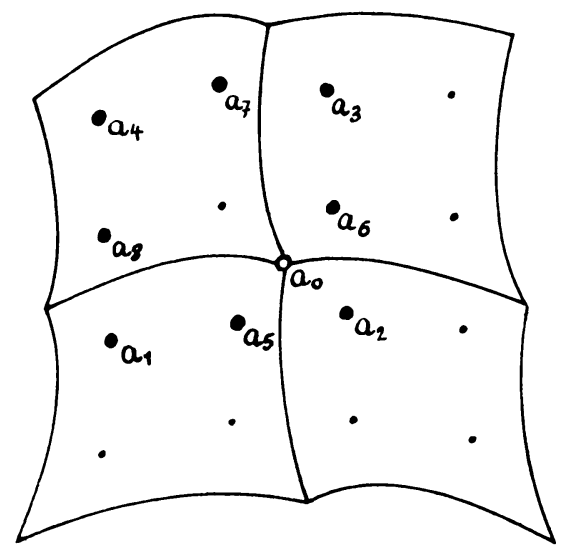

FIGURE 1

way is to use the quadratic isoparametric shape function. We choose eight Gaussian points $a_{j}\left(x_{1}^{j}, x_{2}^{j}\right), j=1, \ldots, 8$ (see Figure 1). The interpolation of the partial derivatives $\partial u_{h} / \partial x_{i}$ at the point $a_{0}=\left(x_{1}^{0}, x_{2}^{0}\right)$ is done by the formula

$$
\sum_{j=1}^{8} \frac{\partial u_{h}\left(x_{1}^{j}, x_{2}^{j}\right)}{\partial x_{i}} N_{j}\left(\xi_{1}^{0}, \xi_{2}^{0}\right) \text {. }
$$

The coordinates $\xi_{1}^{0}, \xi_{2}^{0}$ are computed by solving the system of two nonlinear equations $x_{i}^{0}=\Sigma_{j=1}^{8} x_{i}^{j} N_{j}\left(\xi_{1}, \xi_{2}\right), \quad i=1,2$, by Newton's method. As the initial guess, we choose the point $(0,0)$. The convergence is very fast, and it is entirely sufficient to stop after three iterations.

Computing Center of the Technical University

Obráncủ míru 21

60200 Brno, Czechoslovakia

1. J. BARLOW, “Optimal stress locations in finite element models," Internat. J. Numer. Methods., v. 10, 1976, pp. 243-251.

2. J. H. BRAMBLE \& S. R. HILBERT, "Estimation of linear functionals on Sobolev spaces with application to Fourier transforms and spline interpolation," SIAM J. Numer. Anal., v. 7, 1970, pp. 113-124.

3. J. H. BRAMBLE \& S. R. HILBERT, "Bounds for a class of linear functionals with applications to Hermite interpolation," Numer. Math., v. 16, 1971, pp. 362-369.

4. P. G. CIARLET \& P. A. RAVIART, "The combined effect of curved boundaries and numerical integration in isoparametric finite element methods," The Mathematical Foundations of the Finite Element Method with Applications to Partial Differential Equations (A. K. Aziz, Editor), Academic Press, New York, 1972, pp. 409-474.

5. B. M. IRONS \& A. RAZZAQUE; "Experience with the patch test for convergence of finite elements," The Mathematical Foundations of the Finite Element Method with Applications to Partial Differential Equations (A. K. Aziz, Editor), Academic Press, New York, 1972, pp. 557-587.

6. J. NECAS, Les Méthodes Directes en Théorie des Equations Elliptiques, Academia, Prague, 1967.

7. D. A. VERYARD, Problems Associated with the Convergence of Isoparametric and Mixoparametric Finite Elements, M. Sc. Thesis, University of Wales, 1971.

8. O. C. ZIENKIEWICZ, The Finite Element Method in Engineering Science, McGraw-Hill, London, 1972.

9. M. ZLÁMAL, "Some superconvergence results in the finite element method," Mathematical Aspects of Finite Element Methods, Springer-Verlag, Berlin, Heidelberg, New York, 1977, pp. $351-362$. 